Check for updates

Cite this: RSC Adv., 2021, 11, 24772

\title{
One-step synthesis, characterization and properties of novel hybrid electromagnetic nanomaterials based on polydiphenylamine and Co-Fe particles in the absence and presence of single-walled carbon nanotubes
}

\author{
Sveta Zhiraslanovna Ozkan, (DD *a Galina Petrovna Karpacheva, ${ }^{a}$ \\ Mikhail Nikolaevich Efimov, ${ }^{a}$ Andrey Aleksandrovich Vasilev, ${ }^{a}$ \\ Dmitriy Gennad'evich Muratov, ${ }^{a}$ Valeriy Alekseevich Petrov, ${ }^{a}$ \\ Petr Aleksandrovich Chernavskii ${ }^{\mathrm{ab}}$ and Galina Viktorovna Pankina ${ }^{\mathrm{ab}}$
}

\begin{abstract}
A one-step preparation method for hybrid electromagnetic nanomaterials based on polydiphenylamine (PDPA) and bimetallic Co-Fe particles in the absence and presence of single-walled carbon nanotubes (SWCNT) was proposed. During IR heating of PDPA in the presence of Co(॥) and Fe(III) salts in an inert atmosphere at $T=450-600{ }^{\circ} \mathrm{C}$, the polycondensation of diphenylamine (DPA) oligomers and dehydrogenation of phenyleneamine units of the polymer with the formation of $\mathrm{C}=\mathrm{N}$ bonds and reduction of metals by evolved hydrogen with the formation of bimetallic Co-Fe particles dispersed in a polymer matrix occur simultaneously. When carbon nanotubes are introduced into the reaction system, a nanocomposite material is formed, in which bimetallic Co-Fe particles immobilized on SWCNT are distributed in the matrix of the polymer. According to XRD data, reflection peaks of bimetallic Co-Fe particles at diffraction scattering angles $2 \theta=69.04^{\circ}$ and $106.5^{\circ}$ correspond to a solid solution based on the fCC-Co crystal lattice. According to SEM and TEM data, a mixture of particles with sizes of 8-30 nm and 400-800 nm (Co-Fe/PDPA) and 23-50 nm and 400-1100 nm (Co-Fe/SWCNT/ PDPA) is formed in the nanocomposites. The obtained multifunctional Co-Fe/PDPA and Co-Fe/SWCNT/ PDPA nanomaterials demonstrate good thermal, electrical and magnetic properties. The saturation magnetization of the nanomaterials is $M_{\mathrm{S}}=14.99-31.32 \mathrm{emu} \mathrm{g}^{-1}$ (Co-Fe/PDPA) and $M_{\mathrm{S}}=29.48-48.84$ emu $\mathrm{g}^{-1}$ (Co-Fe/SWCNT/PDPA). The electrical conductivity of the nanomaterials reaches $3.5 \times$ $10^{-3} \mathrm{~S} \mathrm{~cm}^{-1}$ (Co-Fe/PDPA) and $1.3 \mathrm{~S} \mathrm{~cm}^{-1}$ (Co-Fe/SWCNT/PDPA). In an inert medium, at $1000{ }^{\circ} \mathrm{C}$ the residue is $71-77 \%$.
\end{abstract}

Accepted 8th July 2021

DOI: $10.1039 / \mathrm{d} 1 \mathrm{ra03114g}$

rsc.li/rsc-advances electrons along the conjugation chain. ${ }^{4-6}$ The specific electronic structure of polymers with a system of polyconjugation determines their excellent electrophysical, optical and electrochemical properties. ${ }^{7-16}$

The primary advantages of ternary nanocomposites are the synergistic effect between the three components, resulting in excellent electronic, optical, sensing, catalytic, magnetic properties, environmental and thermal stability. ${ }^{17-28}$ Polymer-metalcarbon nanomaterials based on conjugated polymers can be used in magnetic information recording systems, for medical purposes, as electromagnetic radiation absorbing materials, to create sensors and nanoprobes, rechargeable batteries, and other electrochemical devices.

The strategy for the synthesis of ternary systems usually involves two stages. There are two most common approaches to the preparation of ternary nanocomposites. The first way is the in situ oxidative polymerization of aromatic amines in the 
presence of prefabricated binary metal-carbon nanocomposites. $^{29-34}$ The second approach provides the in situ oxidative polymerization of aromatic amines in the presence of carbon nanomaterials followed by the deposition of magnetic nanoparticles on the surface of polymer-carbon nanocomposites. ${ }^{17,35-37}$ Aniline, pyrrole, thiophene and their derivatives are examples of aromatic amines monomers used to synthesize ternary nanocomposites. $\mathrm{Fe}_{3} \mathrm{O}_{4}, \gamma-\mathrm{Fe}_{2} \mathrm{O}_{3}, \alpha-\mathrm{Fe}_{2} \mathrm{O}_{3}$, $\mathrm{Co}_{3} \mathrm{O}_{4}, \mathrm{CoFe}_{2} \mathrm{O}_{4}, \mathrm{FeCoO}$ can be used as magnetic metal oxide nanoparticles. Graphene, reduced graphene oxide (RGO), carbon nanotubes (CNT) can be used as carbonaceous nanofillers. ${ }^{\mathbf{1 - 3 , 3 0 , 3 3 , 3 4 , 3 8 - 4 3}}$ The obtained ternary nanomaterials are superparamagnetic, with saturation magnetization $M_{\mathrm{S}} \sim 19.3-$ 37.2 emu $^{-1}$. $^{29,36,44}$

For instance, $\mathrm{Fe}_{3} \mathrm{O}_{4} / \mathrm{RGO} /$ polyaniline (PANI) ternary nanocomposites were prepared by in situ polymerization of aniline on the $\mathrm{Fe}_{3} \mathrm{O}_{4} /$ RGO surface. ${ }^{30} \mathrm{Fe}_{3} \mathrm{O}_{4}$ nanoparticles with sizes in the range of 5-10 $\mathrm{nm}$ were immobilized on a RGO sheet. The amount of RGO in the nanocomposite was $20-80 \mathrm{wt} \%$. Graphene oxide was reduced with hydrazine. The obtained $\mathrm{Fe}_{3} \mathrm{O}_{4} /$ RGO/PANI nanocomposites are superparamagnetic. Saturation magnetization and residual magnetization decrease, whereas coercive force grows with the increase of the $\mathrm{Fe}_{3} \mathrm{O}_{4} / \mathrm{RGO}$ ratio. The electromagnetic wave absorbing properties of composite materials were measured. The maximum reflection loss was $-36.5 \mathrm{~dB}$ at $7.4 \mathrm{GHz}$ with a thickness of $4.5 \mathrm{~mm}$.

Ternary composites based on $\mathrm{RGO}, \mathrm{Fe}_{3} \mathrm{O}_{4}$ porous nanospheres and PANI were synthesized. ${ }^{45}$ The uniform $\mathrm{Fe}_{3} \mathrm{O}_{4}$ porous nanospheres with the average size of 250-400 nm were grown on the RGO sheets and the PANI layer covered over the surface of $\mathrm{RGO} /$ porous $\mathrm{Fe}_{3} \mathrm{O}_{4}$ binary composite. The ternary composites showed ferromagnetic behavior. As the content of porous $\mathrm{Fe}_{3} \mathrm{O}_{4}$ nanoparticles decreases, the saturation magnetization of the ternary composites decreases, while the coercive force increases. The composites showed excellent microwave absorbing properties. The minimum reflection loss value reached $-29.51 \mathrm{~dB}$ at $14.69 \mathrm{GHz}$ with a thickness of $1.0 \mathrm{~mm}$.

Polypyrrole (PPy) $/ \mathrm{Fe}_{2} \mathrm{O}_{3} / \mathrm{RGO}$ ternary composites were obtained via facile two-step synthesis. Method includes the binary $\mathrm{Fe}_{2} \mathrm{O}_{3} /$ RGO composite preparation under hydrothermal conditions, followed by oxidative polymerization of pyrrole on the $\mathrm{Fe}_{2} \mathrm{O}_{3} /$ RGO surface. Testing of ternary nanocomposites as an electrode material revealed their high specific capacitance of $140 \mathrm{~F} \mathrm{~g}^{-1}$ and excellent capacitance stability of $93 \% .^{46}$

$\mathrm{PPy} / \mathrm{Fe}_{3} \mathrm{O}_{4} / \mathrm{CNT}$ ternary nanocomposites were prepared via oxidative polymerization of pyrrole in the presence of carbon nanotubes decorated with $\mathrm{Fe}_{3} \mathrm{O}_{4}$ nanoparticles. ${ }^{23}$ Nanocomposites have both magnetic loss and dielectric loss resulting in a good electromagnetic wave absorbing properties. The maximum reflection loss value was $-51.67 \mathrm{~dB}$ at $10.2 \mathrm{GHz}$ with a thickness of $3.0 \mathrm{~mm}$.

Ternary nanocomposites based on PANI, graphene and magnetite nanoparticles were synthesized via a two-step method. ${ }^{36}$ Graphene/PANI nanocomposite was prepared via oxidative polymerization of aniline in a dilute solution of sulfuric acid in the presence of graphene oxide reduced with hydrazine. The $\mathrm{Fe}_{3} \mathrm{O}_{4}$ nanoparticles with the size in the range of
5-20 nm were anchored via co-precipitation. The obtained graphene/PANI $/ \mathrm{Fe}_{3} \mathrm{O}_{4}$ nanocomposites are superparamagnetic, with saturation magnetization $M_{\mathrm{S}}$ of $19.3 \mathrm{emu} \mathrm{g}^{-1}$.

Nanocomposites containing PANI, multi-walled carbon nanotubes (MWCNT) and magnetite nanoparticles were prepared via a two-step method. ${ }^{35}$ First, in situ oxidative polymerization of aniline in the presence of MWCNT treated with concentrated nitric acid was performed. Then, the $\mathrm{Fe}_{3} \mathrm{O}_{4}$ nanoparticles modified with aniline dimer were added to the reaction solution. ${ }^{47}$ The resulting $\mathrm{MWCNT} / \mathrm{PANI} / \mathrm{Fe}_{3} \mathrm{O}_{4}$ ternary nanomaterials can be easily dispersed in water and separated under an external magnetic field.

$\mathrm{Fe}_{2} \mathrm{O}_{3} /$ poly(3,4-ethylene-dioxythiophene) (PEDOT)/RGO ternary nanocomposites were synthesized by one-pot method. ${ }^{25}$ The reaction mixture was placed in an autoclave and kept at $170{ }^{\circ} \mathrm{C}$ for $4 \mathrm{~h}$. Then, the mixture was centrifuged, washed with ethanol and water, and dried at $60{ }^{\circ} \mathrm{C}$. An electrochemical sensor based on $\mathrm{Fe}_{2} \mathrm{O}_{3}$ /PEDOT/RGO for the determination of caffeine was developed and applied successfully to the analysis of caffeine in beverage samples.

We have expanded the range of electroactive polymers as part of hybrid polymer-metal-carbon nanocomposites. A simple and effective method to obtain nanocomposite magnetic materials based on poly-3-amine-7-methylamine-2methylphenazine (PAMMP) via oxidative polymerization of 3amine-7-dimethylamine-2-methylphenazine hydrochloride on the surface of magnetite nanoparticles immobilized on singlewalled carbon nanotubes $\left(\mathrm{Fe}_{3} \mathrm{O}_{4} / \mathrm{SWCNT}\right)$ was proposed. ${ }^{48}$ The prepared ternary nanomaterials are electrically conductive, with high saturation magnetization $\left(M_{\mathrm{S}}\right.$ up to $\left.47.24 \mathrm{emu}^{-1}\right)$ and thermal stability.

Hybrid ternary nanomaterials based on polydiphenylamine2-carboxylic acid (PDPAC), $\mathrm{Fe}_{3} \mathrm{O}_{4}$ nanoparticles and SWCNT were obtained for the first time. ${ }^{49}$ Polymer-metal-carbon $\mathrm{Fe}_{3} \mathrm{O}_{4} /$ SWCNT/PDPAC nanocomposites were synthesized via oxidative polymerization of diphenylamine-2-carboxylic acid (DPAC) by two different ways: in an acidic medium and in the interfacial process in an alkaline medium. Electrical conductivity of the nanomaterials depends strongly on the synthesis method as well as on the concentration both of carbon nanotubes and of $\mathrm{Fe}_{3} \mathrm{O}_{4}$ nanoparticles. Depending on the synthesis method, the electrical conductivity of the nanocomposites reaches $9.6 \times$ $10^{-7} \mathrm{~S} \mathrm{~cm}^{-1}$ and $3.3 \times 10^{-3} \mathrm{~S} \mathrm{~cm}^{-1}$. Higher electrical conductivity of nanomaterials synthesized in an acidic medium is due to the doping of the polymer matrix that occurs during the synthesis of nanocomposites. Regardless of the preparation method, the $\mathrm{Fe}_{3} \mathrm{O}_{4} / \mathrm{SWCNT} / \mathrm{PDPAC}$ nanomaterials are superparamagnetic $\left(\kappa_{\pi}=M_{\mathrm{R}} / M_{\mathrm{S}}=0\right)$. The saturation magnetization $M_{\mathrm{S}}$ depends on the content of magnetite nanoparticles and reaches 31.6-39.4 emu $^{-1}$ depending on the synthesis method of nanocomposites. At the same time, the $\mathrm{Fe}_{3} \mathrm{O}_{4} / \mathrm{SWCNT} / \mathrm{PDPAC}$ nanomaterials prepared in the interfacial process in an alkaline medium by one-pot method form high stability magnetic fluids in ethanol. ${ }^{\mathbf{5 0}}$

The analysis of the literature data showed that polymermetal-carbon composites have a high potential for practical use in industry, medicine, energy and human life due to their 
multifunctionality. However, their practical application is hindered by the complexity of the multi-stage synthesis of such ternary nanomaterials. The problem can be solved by developing a facile one-stage environmentally friendly synthesis method. According to our knowledge, no comprehensive systematic research has been conducted in this area. Ternary PEDOT/GO/ $\mathrm{Fe}_{2} \mathrm{O}_{3}$ nanocomposites were obtained by electropolymerization of 3,4-ethylene-dioxythiophene monomer on a substrate in the presence of GO and iron sulfate. ${ }^{26}$ The study of electrochemical behavior of $\mathrm{PEDOT} / \mathrm{GO} / \mathrm{Fe}_{2} \mathrm{O}_{3}$ nanocomposites showed a high specific electrochemical capacity $\left(221 \mathrm{~F} \mathrm{~g}^{-1}\right)$ at a charge-discharge rate of $25 \mathrm{mV} \mathrm{s}^{-1}$ and long-term stability performance even after 1000 cycles owing to the high mechanical strength provided by GO. These performances make PEDOT/GO/ $\mathrm{Fe}_{2} \mathrm{O}_{3}$ composites as promising material for supercapacitor. However, the fact that the resulting material exists on a substrate significantly limits the scope of its practical application. The task of developing facile and energy-saving methods for obtaining ternary nanomaterials remains urgent.

It should be noted that to obtain ternary nanomaterials, oxidative polymerization is usually carried out on the surface of prefabricated magnetic nanoparticles $\left(\mathrm{Fe}_{3} \mathrm{O}_{4}, \gamma-\mathrm{Fe}_{2} \mathrm{O}_{3}, \alpha-\mathrm{Fe}_{2} \mathrm{O}_{3}\right.$, $\left.\mathrm{Co}_{3} \mathrm{O}_{4}, \mathrm{CoFe}_{2} \mathrm{O}_{4}, \mathrm{FeCoO}\right)$. Metal oxide nanoparticles are characterized by not too high magnetic characteristics depending on the synthesis conditions. Besides, the high surface energy of such nanoparticles results in their strong tendency for aggregation. One of the ways to prevent the aggregation of metal nanoparticles during synthesis is to stabilize them in a polymer matrix. The difficulty of the inclusion of other magnetic nanoparticles in the composition of nanocomposites is attributed to the need to develop a complex synthetic strategy that would make it possible to expand the range of magnetic nanoparticles in the composition of polymer-metal-carbon nanocomposites. It is more preferable to use bimetallic magnetic nanoparticles with higher values of magnetic characteristics, such as Co-Fe, $\mathrm{Ni}-\mathrm{Fe}$ or $\mathrm{Co}-\mathrm{Ni}$, as a magnetic component in nanocomposites. ${ }^{51-54}$

Earlier we have synthesized hybrid magnetic nanocomposites based on polyphenoxazine (PPOA) and bimetallic Co-Fe nanoparticles under IR heating. ${ }^{55}$ The prepared metalpolymer nanomaterials are thermally stable and superparamagnetic. Saturation magnetization of $\mathrm{Co}-\mathrm{Fe} / \mathrm{PPOA}$ nanocomposites reaches $M_{\mathrm{S}}=27.3 \mathrm{emu} \mathrm{g}^{-1}$. As can be seen from Table 1, the use of iron or cobalt oxides as magnetic nanoparticles leads to low values of saturation magnetization and to an increase in the coercive force. ${ }^{56-59}$

We have previously shown that the size, shape, and phase state of the resulting particles depend on the nature of the polymer matrix. An important role belongs to the thermal stability of the polymer, the presence of functional groups capable of complexation with ions of metals, because acidic residues of salts are carried away by the argon flow under IR heating, and also the presence of a sufficient amount of hydrogen in the polymer structure capable of reducing metals during dehydrogenation. This was shown in the preparation of metal-polymer nanocomposites based on PPOA and polydiphenylamine (PDPA) in the presence of salts $\left(\mathrm{FeCl}_{3} \cdot 6 \mathrm{H}_{2} \mathrm{O}\right.$ or $\left.\mathrm{Co}\left(\mathrm{OOCCH}_{3}\right)_{2} \cdot 4 \mathrm{H}_{2} \mathrm{O}\right)$, when various cobalt- and iron-containing nanoparticles of different sizes and shapes were formed. ${ }^{56-60}$ Under the same synthesis conditions, IR heating of PDPA in the presence of $\mathrm{Co}\left(\mathrm{OOCCH}_{3}\right)_{2} \cdot 4 \mathrm{H}_{2} \mathrm{O}$ leads to the formation of spherical $\alpha$-Co nanoparticles with a hexagonal close-packed lattice and $\beta$-Co nanoparticles with a face-centered cubic lattice, their size ranging $2<d<8 \mathrm{~nm}$. In the case of PPOA, only spherical $\beta$-Co nanoparticles are formed, their size ranging $4<$ $d<14 \mathrm{~nm}$. In the presence of $\mathrm{FeCl}_{3} \cdot 6 \mathrm{H}_{2} \mathrm{O}$ in PPOA, ironcontaining nanoparticles $\mathrm{Fe}_{2} \mathrm{O}_{3}, \mathrm{Fe}_{3} \mathrm{O}_{4}, \mathrm{FeO}, \alpha$-Fe, $\gamma$-Fe, as well as $\mathrm{Fe}_{3} \mathrm{~N}, \mathrm{Fe}_{4} \mathrm{~N}$ and $\mathrm{Fe}_{3} \mathrm{C}$ are formed, their size and shape depend on the synthesis conditions.

PDPA, an aromatic derivative of PANI, is characterized by better solubility in organic solvents than PANI, redox activity, electrical and optical properties and high thermal stability. PDPA was synthesized by chemical oxidative polymerization or under conditions of electrosynthesis. ${ }^{61-65}$ Due to the low electrical conductivity, PDPA has not attracted much attention of researchers until recently. As part of binary nanocomposites with carbon nanotubes or polyvinyl fluoride, PDPA has been studied to create sensors and rechargeable lithium batteries, in particular as an additive to a polymer electrolyte. ${ }^{\mathbf{6 6 - 6 8}}$ However, PDPA has a high intrinsic theoretical specific electrochemical capacity ( $160 \mathrm{~W} \mathrm{~h} \mathrm{~kg}^{-1}$ ) and can be considered as an independent cathode material for rechargeable batteries. Dedoped PDPA was proposed as a highly promising cathode material for lithium and potassium based dual-ion batteries. PDPA showed a record high value of energy density with an active material loading of $80 \%$ ( $418 \mathrm{~W} \mathrm{~h} \mathrm{~kg}^{-1}$, calculated for full cathode mass). It outperforms significantly the characteristics of all known polymeric cathodes for dual-ion batteries. ${ }^{69}$

The low electrical conductivity of PDPA proved to be in demand in the production of electrorheological suspensions. Electrorheological suspension based on silica/PDPA microspheres was prepared without dedoping step due to quite low conductivity of PDPA. Novel semiconductive nanoscale incapsulated spherical particles with core-shell structure were prepared via chemical oxidative polymerization DPA in the presence of silica particles. PDPA shell thickness is of

Table 1 Magnetic properties of metal-polymer nanomaterials

\begin{tabular}{|c|c|c|c|c|c|c|}
\hline Nanomaterials & Me phase composition & $H_{\mathrm{C}}$, Oe & $M_{\mathrm{S}}$, emu g $^{-1}$ & $M_{\mathrm{R}}$, emu g $^{-1}$ & $M_{\mathrm{R}} / M_{\mathrm{S}}$ & References \\
\hline $\mathrm{Co}-\mathrm{Fe} / \mathrm{PPOA}$ & Co-Fe & 55 & 27.28 & 0.7 & 0.025 & 55 \\
\hline $\mathrm{Fe}_{3} \mathrm{O}_{4} / \mathrm{PPOA}$ & $\mathrm{Fe}_{3} \mathrm{O}_{4}$ & 269 & 19.35 & 5.3 & 0.274 & 56 and 57 \\
\hline CoO/PDPA & $\mathrm{CoO}$ & 142 & 0.984 & 0.076 & 0.08 & 59 \\
\hline
\end{tabular}


approximately $30 \mathrm{~nm}$. Superior sensitivity and reversibility were observed by the on-off test. ${ }^{70}$

In this study, a one-step synthesis method for metal-polymer and polymer-metal-carbon electromagnetic nanomaterials based on PDPA and bimetallic Co-Fe particles was developed for the first time. The nanocomposites were prepared by IR heating of precursors based on PDPA, cobalt(II) and iron(III) salts in an inert atmosphere. SWCNT were used as a carbonaceous component. A comparative analysis of the structure, morphology, thermal, magnetic, and electrical properties of CoFe/PDPA and Co-Fe/SWCNT/PDPA nanocomposites depending on the synthesis conditions was done.

\section{Experimental}

\subsection{Materials}

Diphenylamine (DPA) (analytical grade) was purified by double recrystallization from isopropyl alcohol, and ammonium persulfate (analytical grade) was purified by recrystallization from distilled water. Isopropyl alcohol (extra pure grade), toluene (analytical grade), aqueous ammonia (reagent grade), DMFA (Acros Organics, 99\%), as well as $\mathrm{Co}\left(\mathrm{OOCCH}_{3}\right)_{2} \cdot 4 \mathrm{H}_{2} \mathrm{O}$ (pure grade) and iron(III) chloride (pure grade) were used without additional purification. Aqueous solutions of reagents were prepared with the use of distilled water. SWCNT from Carbon Chg, Ltd. were produced using electric arc discharge technique with a $\mathrm{Ni} / \mathrm{Y}$ catalyst $(d=1.4-1.6 \mathrm{~nm}, l=0.5-1.5 \mu \mathrm{m})$.

\subsection{Synthesis of polydiphenylamine}

Polydiphenylamine (PDPA) was synthesized under conditions of chemical oxidative polymerization in situ in the interfacial process according to the method developed by the authors in. ${ }^{62}$ To synthesize PDPA in the interfacial process, $0.2 \mathrm{~mol} \mathrm{~L}^{-1}(6.0 \mathrm{~g})$ of DPA were dissolved in an organic solvent-toluene $(90 \mathrm{~mL})$, whereas $0.25 \mathrm{~mol} \mathrm{~L}^{-1}(10.26 \mathrm{~g})$ of ammonium persulfate and $1.0 \mathrm{~mol} \mathrm{~L}^{-1}(15.0 \mathrm{~mL})$ of hydrochloric acid were dissolved in distilled water $(75.0 \mathrm{~mL})$. The volume ratio of the aqueous and organic phases is $1: 1$. Solutions of the organic and aqueous phases were mixed immediately, without gradual dosing of reagents. The process was carried out under vigorous stirring using an Ika Werke RW 16 Basic electronic overhead stirrer in a narrow cylindrical round-bottom two-necked flask (to increase the stirring efficiency) at $0{ }^{\circ} \mathrm{C}$ for $4 \mathrm{~h}$. When the synthesis was completed, the reaction mixture was precipitated in a fivefold excess of isopropyl alcohol $(400 \mathrm{~mL})$. The obtained product was filtered off and washed repeatedly with distilled water to remove residual reagents. The neutralization of PDPA was carried out in a $3 \%$ solution of $\mathrm{NH}_{4} \mathrm{OH}(200 \mathrm{~mL})$ for $24 \mathrm{~h}$; after that, the polymer was filtered off and washed repeatedly in the excess of distilled water until neutral reaction, and then vacuum-dried over $\mathrm{KOH}$ to constant weight. The PDPA yield is $5.15 \mathrm{~g}$ (85.8\%).

\subsection{Synthesis of Co-Fe/PDPA}

The following method was used to obtain Co-Fe/PDPA. ${ }^{71}$ A cosolution of PDPA, $\mathrm{Co}\left(\mathrm{OOCCH}_{3}\right)_{2} \cdot 4 \mathrm{H}_{2} \mathrm{O}$ and $\mathrm{FeCl}_{3} \cdot 6 \mathrm{H}_{2} \mathrm{O}$ in DMFA was prepared in a crystallization dish. The concentration 
of PDPA in the DMFA solution was $2 \mathrm{wt} \%$, the content of cobalt(II) $[\mathrm{Co}]=3-10 \mathrm{wt} \%$, and iron(III) $[\mathrm{Fe}]=5-22 \mathrm{wt} \%$ relative to the polymer weight. The ratios of metals are given in Table 2. After the solvent was removed at $T=60-85{ }^{\circ} \mathrm{C}$, the precursor consisting of PDPA and cobalt(II) acetate and iron(III) chloride salts was IR-heated with an automated IR heating unit in an Ar atmosphere at different sample temperatures in the range of $T$ $=400-600{ }^{\circ} \mathrm{C}$ for $2-10 \mathrm{~min}$. The heating rate was $50{ }^{\circ} \mathrm{C} \mathrm{min}^{-1}$. Depending on the selected synthesis conditions, the Co-Fe/ PDPA yield is 58.3-76.0\% (Table 2).

The IR heating unit is a laboratory quartz tube IR furnace..$^{72,73}$ The halogen lamps (total maximum power of $24 \mathrm{~kW}$ ) located outside of quartz reactor were implemented as a radiation source. The maximum wavelength of emission of these IR tubes is in the range of $0.85-1.2 \mu \mathrm{m}$. The rectangular graphite box with the samples covered with a graphite lid was placed in quartz reactor. The heating temperature was measured by a chromel-alumel thermocouple fixed in a similar graphite box next to the sample one and was regulated by the temperature controller via IR radiation intensity adjustment.

\subsection{Preparation of Co-Fe/SWCNT/PDPA}

To synthesize Co-Fe/SWCNT/PDPA a co-solution of PDPA, cobalt(II) acetate $\mathrm{Co}\left(\mathrm{OOCCH}_{3}\right)_{2} \cdot 4 \mathrm{H}_{2} \mathrm{O}$ and iron(III) chloride $\mathrm{FeCl}_{3} \cdot 6 \mathrm{H}_{2} \mathrm{O}$ in DMFA containing SWCNT was prepared. ${ }^{74}$ The concentration of PDPA in the DMFA solution was $2 \mathrm{wt} \%$, the content of carbon nanotubes was [SWCNT] $=3$ and $10 \mathrm{wt} \%$, cobalt(II) $[\mathrm{Co}]=3-10 \mathrm{wt} \%$, and iron(III) $[\mathrm{Fe}]=5-22 \mathrm{wt} \%$ relative to the polymer weight. The ratios of metals are given in Table 2 . After the solvent was removed at $T=60-85{ }^{\circ} \mathrm{C}$, the precursor consisting of PDPA, SWCNT, and cobalt(II) acetate and iron(III) chloride salts was IR-heated in an Ar atmosphere at various sample temperatures in the range of $T=400-600{ }^{\circ} \mathrm{C}$ for $2-$ $10 \mathrm{~min}$. Depending on the selected synthesis conditions, the Co-Fe/SWCNT/PDPA yield is $63.7-77.4 \%$ (Table 2).

\subsection{Characterization}

The metal content in the nanocomposites was measured quantitatively by an inductively coupled plasma atomic emission spectroscopy method (ICP-AES) using a Shimadzu ICP emission spectrometer (ICPE-9000) (Kyoto, Japan).

Attenuated Total Reflection (ATR) FTIR spectra of the samples in the attenuated total reflectance mode were recorded using a HYPERION-2000 IR microscope (Bruker, Karlsruhe, Germany) coupled with the Bruker IFS 66v FTIR spectrometer (Karlsruhe, Germany) in the range of $600-4000 \mathrm{~cm}^{-1}$ (150 scans, ZnSe crystal, resolution of $2 \mathrm{~cm}^{-1}$ ).

$\mathrm{X}$-ray diffraction study was performed in ambient atmosphere using a Difray-401 X-ray diffractometer (Scientific Instruments Joint Stock Company, Saint-Petersburg, Russia) with Bragg-Brentano focusing on $\mathrm{CrK}_{\alpha}$ radiation, $\lambda=0.229 \mathrm{~nm}$.

An electron microscopic study was performed using a JEM2100 transmission electron microscope (accelerating voltage of $200 \mathrm{kV}$ ) (JEOL, Akishima, Tokyo, Japan) and a Hitachi TM 3030 scanning electron microscope (Hitachi High-Technologies Corporation, Fukuoka, Japan) with magnification up to 30000 and $30 \mathrm{~nm}$ resolution.

Thermogravimetric analysis (TGA) was performed on a Mettler Toledo TGA/DSC1 (Giessen, Germany) in the dynamic mode in the range of $30-1000{ }^{\circ} \mathrm{C}$ in air and in the argon flow. The weight of the samples was $100 \mathrm{mg}$, the heating rate was $10{ }^{\circ} \mathrm{C} \mathrm{min}{ }^{-1}$, and the argon flow velocity was $10 \mathrm{~mL} \mathrm{~min}{ }^{-1}$. Calcined aluminum oxide was used as a reference. The samples were analyzed in an $\mathrm{Al}_{2} \mathrm{O}_{3}$ crucible.

Differential Scanning Calorimetry (DSC) was performed on a Mettler Toledo DSC823 ${ }^{\mathrm{e}}$ calorimeter (Giessen, Germany). The samples were heated at the rate of $10{ }^{\circ} \mathrm{C} \mathrm{min}^{-1}$ in the nitrogen atmosphere, with the nitrogen flow rate of $70 \mathrm{~mL} \mathrm{~min}^{-1}$. The measurement results were processed with the service program STARe supplied with the device.

The ac conductivity was measured with an E7-20 precision LCR-meter (MC Meratest, Moscow, Russia) in the frequency range of $25.0 \mathrm{~Hz}$ to $1.0 \mathrm{MHz}$.

A vibration magnetometer was used to study the magnetic characteristics of the systems. The cell of the vibration magnetometer was designed as a flow quartz microreactor, which made it possible to study chemical transformations in the in situ mode. ${ }^{75}$ Specific magnetization depending on the

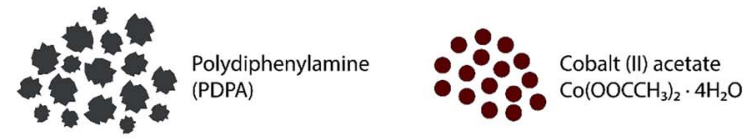
(SWCNT)
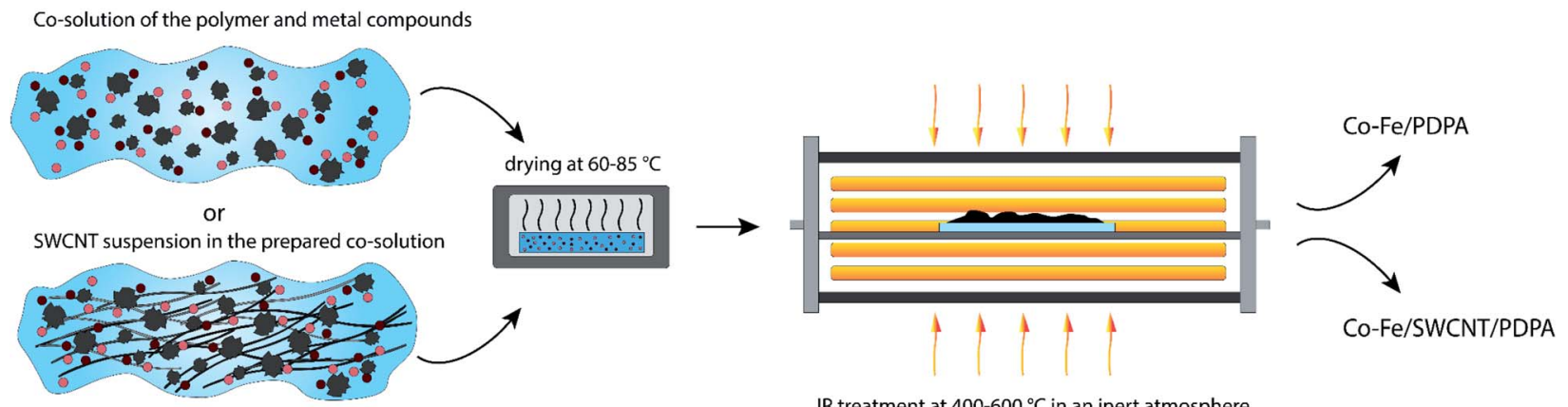

IR treatment at $400-600^{\circ} \mathrm{C}$ in an inert atmosphere

Fig. 1 Synthesis scheme of the Co-Fe/PDPA and Co-Fe/SWCNT/PDPA nanomaterials. 
magnetic field value was measured; magnetic characteristics of the samples at room temperature were determined.

\section{Results and discussion}

\subsection{Characterization of nanomaterials}

A one-step method was proposed to synthesize novel hybrid nanomaterials based on PDPA and bimetallic Co-Fe particles in the absence and presence of SWCNT. PDPA synthesized in the interfacial process for the first time $e^{62,63}$ is an aromatic polyamine with diphenylene units divided by amine groups and has the following structure: correspond to the stretching vibrations of $\nu_{\mathrm{C}-\mathrm{C}}$ bonds in aromatic rings (Fig. 2). As the synthesis temperature rises, there is a decrease in the intensity of the absorption bands at 3380 and $3020 \mathrm{~cm}^{-1}$, corresponding to the stretching vibrations of $\nu_{\mathrm{N}-\mathrm{H}}$ and $\nu_{\mathrm{C}-\mathrm{H}}$ bonds in phenyleneamine structures.

It was found that IR heating of precursors from PDPA, cobalt(II) acetate and iron(III) chloride salts in the absence and presence of SWCNT results in the growth of the polymer chain due to the polycondensation reaction of crystalline DPA oligomers contained in PDPA. This is confirmed by FTIR spectroscopy and XRD data. In the ATR FTIR spectra of Co-Fe/PDPA and $\mathrm{Co}-\mathrm{Fe} / \mathrm{SWCNT/PDPA}$, an increase in the intensity of the

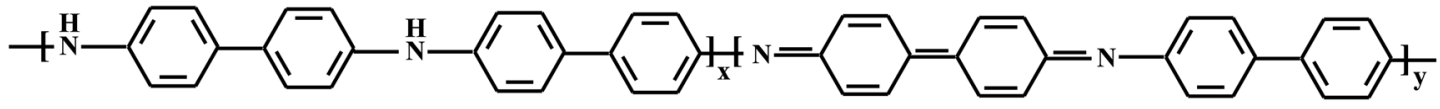

Molecular weight of the polymer reached $M_{\mathrm{w}}=(9-11) \times 10^{3}$. PDPA was chosen due to the simplicity of its synthesis under the conditions of oxidative polymerization and to the stability of the polymer under operating conditions, as well as its high thermal stability (up to $450{ }^{\circ} \mathrm{C}$ in air and up to $600-650{ }^{\circ} \mathrm{C}$ in an inert atmosphere $\left.^{64}\right)$.

A specific feature of the selected polymer is that when PDPA is heated in the presence of metal salts, further growth of the polymer chain occurs due to the polycondensation reaction of crystalline diphenylamine oligomers, as well as dehydrogenation of phenyleneamine units with the formation of $\mathrm{C}=\mathrm{N}$ bonds included in the general system of conjugated double bonds. At the same time, the molecular weight of the polymer increases.

Nanocomposites were synthesized via chemical transformations of PDPA containing cobalt(II) acetate $\mathrm{Co}\left(\mathrm{OOCCH}_{3}\right)_{2} \cdot 4 \mathrm{H}_{2} \mathrm{O}$ and iron(III) chloride $\mathrm{FeCl}_{3} \cdot 6 \mathrm{H}_{2} \mathrm{O}$ salts in the absence and presence of SWCNT in an inert atmosphere under IR heating. As a result, hybrid metal-polymer and polymer-metal-carbon nanocomposites are formed, which are black powders insoluble in organic solvents. Fig. 1 shows a synthesis scheme of the Co-Fe/PDPA and Co-Fe/SWCNT/ PDPA nanomaterials.

The formation of hybrid nanocomposites was confirmed by FTIR spectroscopy and X-ray diffraction analysis (XRD), transmission (TEM) and scanning (SEM) electron microscopy.

The fact that ATR FTIR spectra of Co-Fe/PDPA and Co-Fe/ SWCNT/PDPA retain all the main absorption bands typical of the chemical structure of PDPA confirms the absence of polymer matrix degradation processes during IR heating. FTIR spectroscopy shows that IR heating of precursors leads to dehydrogenation of PDPA phenyleneamine units with the formation of $\mathrm{C}=\mathrm{N}$ bonds incorporated into the general conjugation system. The formation of $\mathrm{C}=\mathrm{N}$ bonds is evidenced by the shift and broadening of the bands at 1593 and $1490 \mathrm{~cm}^{-1}$, that absorption band at $810 \mathrm{~cm}^{-1}$, caused by non-planar deformation vibrations of $\delta_{\mathrm{C}-\mathrm{H}} 4,4 /$-substituted benzene rings, ${ }^{62}$ indicates the growth of the PDPA polymer chain. At the same time,
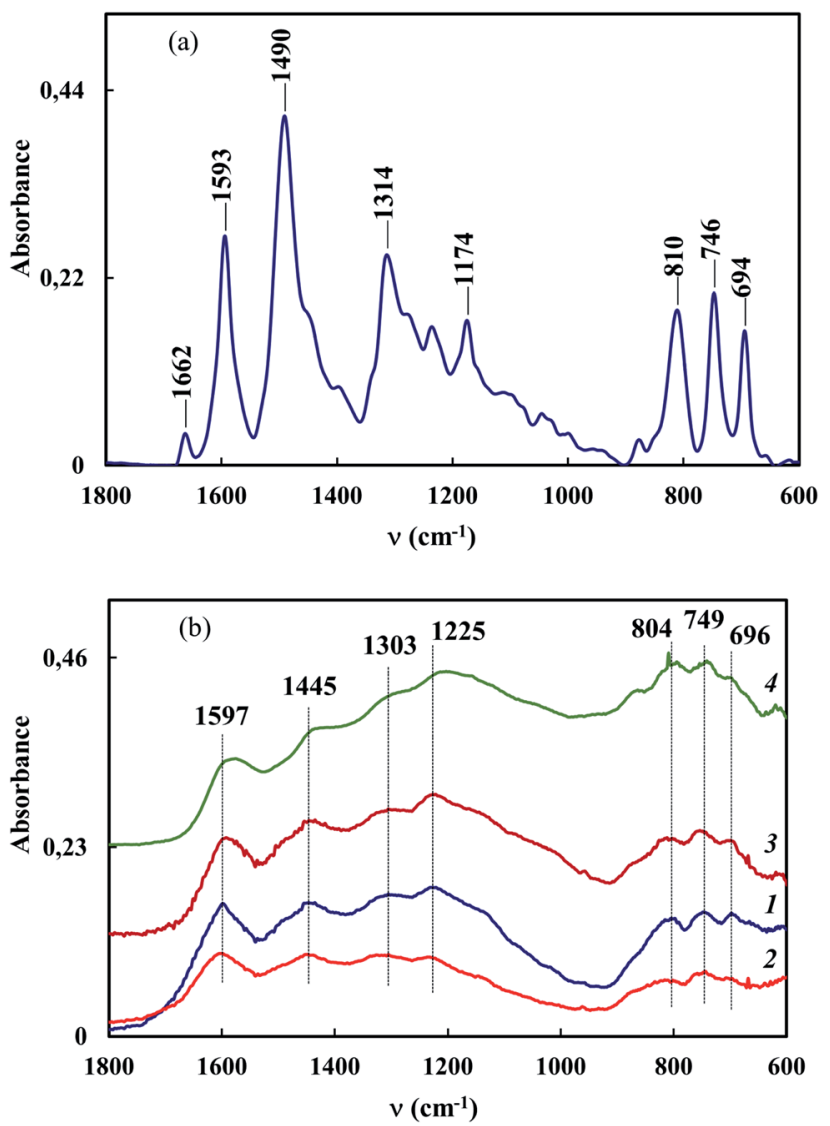

Fig. 2 ATR FTIR spectra of PDPA (a), $\mathrm{Co}, \mathrm{Fe}_{3} \mathrm{O}_{4} / \mathrm{PDPA}$ (1b), Co-Fe/ PDPA (2b), $\mathrm{Co}, \mathrm{Fe}_{3} \mathrm{O}_{4} / \mathrm{SWCNT/PDPA}$ (3b) and Co-Fe/SWCNT/PDPA (4b), prepared at $400(1$ and 3$)$ and $450{ }^{\circ} \mathrm{C}(2$ and 4$),[\mathrm{Co}]=5 \mathrm{wt} \%$ and $[\mathrm{Fe}]=10 \mathrm{wt} \%$, [SWCNT] $=10 \mathrm{wt} \%$. 
the intensity of the absorption band at $694 \mathrm{~cm}^{-1}$ of the monosubstituted phenyl ring decreases, i.e. the number of end groups of the polymer drops considerably (Fig. 2). In addition, the diffraction pattern of the initial PDPA subjected to IR heating at $600{ }^{\circ} \mathrm{C}$ and the diffraction patterns of nanocomposites lack reflection peaks at scattering angles $2 \theta=20$ $50^{\circ}$, which characterize the crystalline low-molecular phase. After processing neat PDPA at $600{ }^{\circ} \mathrm{C}$, a wide halo is observed in this area, which confirms the amorphous structure of the polymer (Fig. 3a). Table 3 shows XRD data of the crystalline lowmolecular phase of neat PDPA.
The PDF-2 database of the International Centre for Diffraction Data (ICDD) was used to identify the phases. ${ }^{76}$

At the same time, according to the elemental analysis data, the hydrogen content in the nanocomposites decreases in comparison with PDPA and depends on the IR heating temperature and the quantitative content of cobalt and iron salts in the precursor (Table 4). For instance, in the presence of $\mathrm{Co}$ (II) and Fe(III) salts along with SWCNT, the hydrogen content in PDPA decreases from $5.8 \%$ for the neat polymer to $1.3 \%$ when the IR heating temperature is increased to $600{ }^{\circ} \mathrm{C}$. Elimination of hydrogen can occur both due to the dehydrogenation
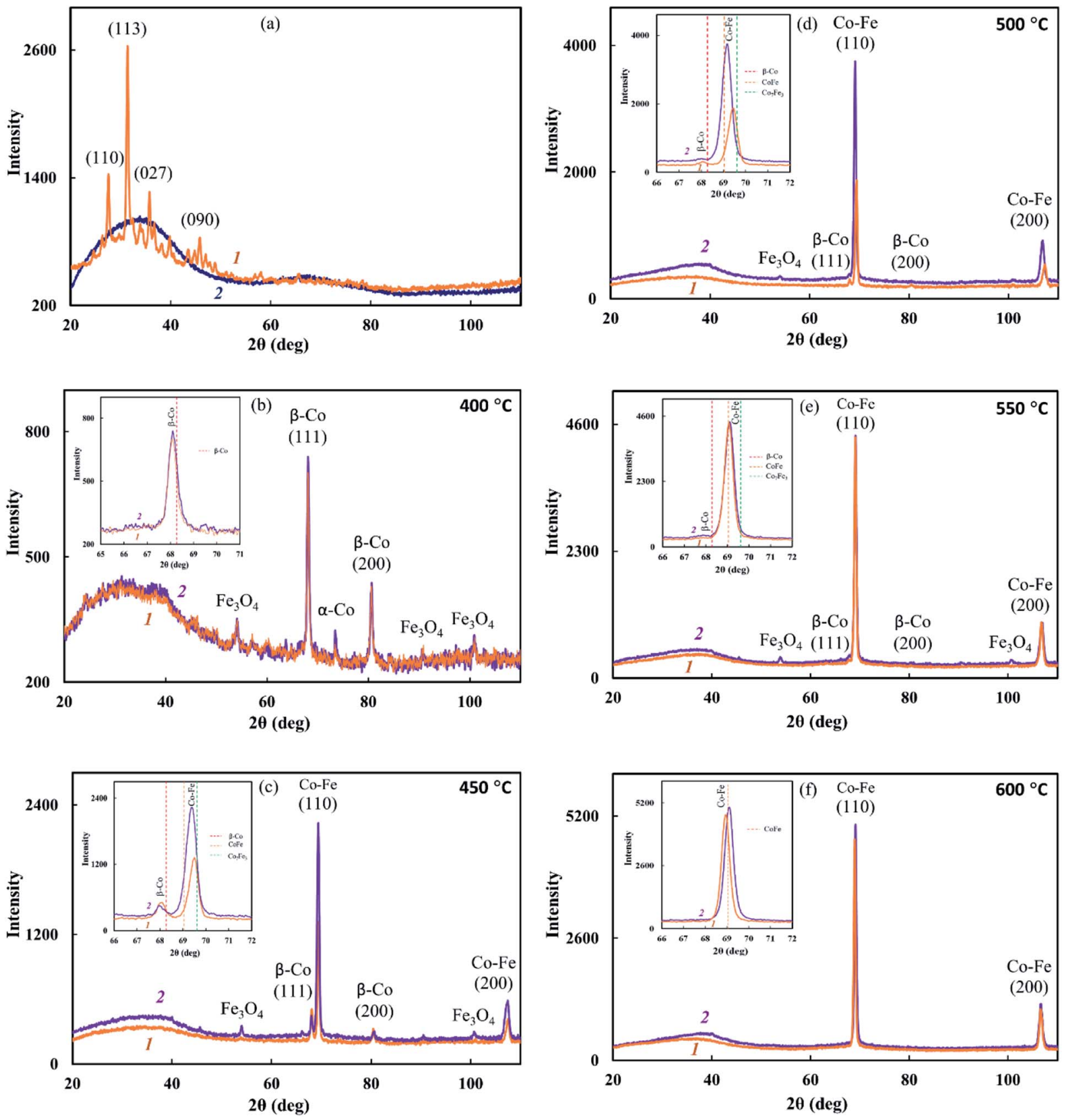

Fig. 3 XRD patterns of PDPA (1a) and PDPA at $600{ }^{\circ} \mathrm{C}$ (2a), $\mathrm{Co}, \mathrm{Fe}_{3} \mathrm{O}_{4} / \mathrm{PDPA}$ (1b), Co, $\mathrm{Fe}_{3} \mathrm{O}_{4} / \mathrm{SWCNT} / \mathrm{PDPA}(2 \mathrm{~b}), \mathrm{Co}-\mathrm{Fe} / \mathrm{PDPA}$ (1c-1f) and $\mathrm{Co}-\mathrm{Fe} /$ SWCNT/PDPA (2c-2f), prepared at $400{ }^{\circ} \mathrm{C}$ (b), $450-600{ }^{\circ} \mathrm{C}(\mathrm{c}-\mathrm{f}),[\mathrm{Co}]=5 \mathrm{wt} \%$ and $[\mathrm{Fe}]=10 \mathrm{wt} \%$, [SWCNT] $=10 \mathrm{wt} \%$. The inserts show line diagrams for $\beta$-Co and Co-Fe. 
Table 3 XRD data of the crystalline low-molecular phase of neat PDPA

\begin{tabular}{llll}
\hline $2 \theta,^{\circ}$ & $d, \AA$ & $h k l$ & Intensity \\
\hline 26.34 & 5.027 & 025 & 32 \\
27.57 & 4.806 & 110 & 54 \\
31.39 & 4.235 & 113 & 100 \\
32.4 & 4.101 & 123 & 39 \\
35.77 & 3.730 & 027 & 48 \\
36.73 & 3.636 & 105 & 37 \\
39.85 & 3.361 & 018 & 32 \\
45.85 & 2.941 & 090 & 32 \\
47.85 & 2.825 & 173 & 23 \\
48.84 & 2.771 & 147 & 23 \\
\hline
\end{tabular}

reaction of phenyleneamine units and due to the polycondensation reaction of DPA oligomers. The evolved hydrogen contributes to the reduction of metals.

The reduction of metals accompanied by the formation of bimetallic Co-Fe particles was confirmed by XRD. The diffraction patterns of nanocomposites show reflection peaks of bimetallic Co-Fe particles at diffraction scattering angles $2 \theta=$ $69.04^{\circ}, 106.5^{\circ}$ (Fig. 3c-f) that correspond to a solid solution based on the fcc-Co crystal lattice. ${ }^{55}$ The inserts show line diagrams for $\beta$-Co and Co-Fe.

Based on the XRD results shown in Fig. 3, the formation of a Co-Fe solid solution can be represented as follows. At $400{ }^{\circ} \mathrm{C}$, the fcc phase of the Co-Fe solid solution on the basis of the $\beta$-Co crystal lattice with the cobalt content of $75-100 \%$ is formed. Then, as iron is reduced and dissolved in the fcc lattice, a polymorphic transformation of the fcc lattice of the solid solution into the bcc lattice with a predominant cobalt content occurs. Upon the complete reduction of iron and the interaction of a large number of metal particles with each other, particles of the $\mathrm{Co}-\mathrm{Fe}$ solid solution are formed with the metal content close to $\mathrm{Fe}: \mathrm{Co}=50: 50$.

Comparison of the values of Miller indices of interplanar distances in the $\mathrm{Co}-\mathrm{Fe}$ and $\beta$-Co phases with the database shows the formation of a solid solution during the synthesis of nanocomposites. According to the database Miller indices, interplanar distances are: $d_{110}=0.2020 \mathrm{~nm}(\mathrm{Co}-\mathrm{Fe}), d_{111}=$ $0.2040 \mathrm{~nm}(\beta-\mathrm{Co}), d_{200}=0.1429 \mathrm{~nm}(\mathrm{Co}-\mathrm{Fe})$ and $0.1770 \mathrm{~nm}(\beta-$ Co). As seen from Table 2, regardless of the synthesis conditions of nanomaterials, the interplanar distances of the $\beta$-Co phase turned out to be larger in comparison with the tabular data, which indicates the dissolution of iron in the $\beta$-Co lattice.

According to SEM data, not only spherical, but also rectangular particles are formed in the Co-Fe/PDPA and $\mathrm{Co}-\mathrm{Fe} /$ SWCNT/PDPA nanocomposites (Fig. 4). Fig. 5 shows TEM images of nanocomposites at different magnifications. A mixture of particles with sizes of 8-30 nm and 400-800 nm (Co$\mathrm{Fe} / \mathrm{PDPA}$ ) and $23-50 \mathrm{~nm}$ and $400-1100 \mathrm{~nm}$ (Co-Fe/SWCNT/ PDPA) is formed in nanocomposites. The formation of larger Co-Fe particles in the Co-Fe/SWCNT/PDPA nanocomposites is apparently due to the interaction of metal ions with the SWCNT surface during the formation of particles, which leads to a slower reduction of metals. Depending on the synthesis conditions, small peaks of $\mathrm{Fe}_{3} \mathrm{O}_{4}$ and $\beta$-Co remain on diffraction patterns of $\mathrm{Co}-\mathrm{Fe} / \mathrm{SWCNT} / \mathrm{PDPA}$ nanocomposites (Fig. 3).

Thus, at IR heating of precursors from PDPA, cobalt(II) acetate and iron(III) chloride salts in the absence and presence of SWCNT in an inert atmosphere at a sample temperature $T=$ $450-600{ }^{\circ} \mathrm{C}$ the following reactions occur simultaneously: the growth of the PDPA polymer chain due to the polycondensation reaction of crystalline oligomers of DPA; dehydrogenation of phenyleneamine units with the formation of $\mathrm{C}=\mathrm{N}$ bonds; reduction of metals due to the evolved hydrogen with the formation of bimetallic $\mathrm{Co}-\mathrm{Fe}$ particles. This results in the formation of nanostructured Co-Fe/PDPA and Co-Fe/SWCNT/ PDPA materials containing particles of $\mathrm{Co}-\mathrm{Fe}$ solid solution dispersed in a polymer matrix, either free or immobilized on SWCNT.

The fundamental novelty of the proposed approach is determined by the fact that in a self-organizing system within one stage under IR heating conditions, hybrid metal-polymer and polymer-metal-carbon nanocomposite materials are formed with a structure that contains bimetallic particles, free or anchored onto SWCNT, dispersed in the polymer PDPA matrix. Also, the method makes it possible to obtain bimetallic particles directly during the synthesis of nanocomposites, without subjecting the polymer matrix to degradation.

Table 4 Inductively coupled plasma atomic emission spectroscopy (ICP-AES) data of nanocomposites and elemental analysis data of PDPA component

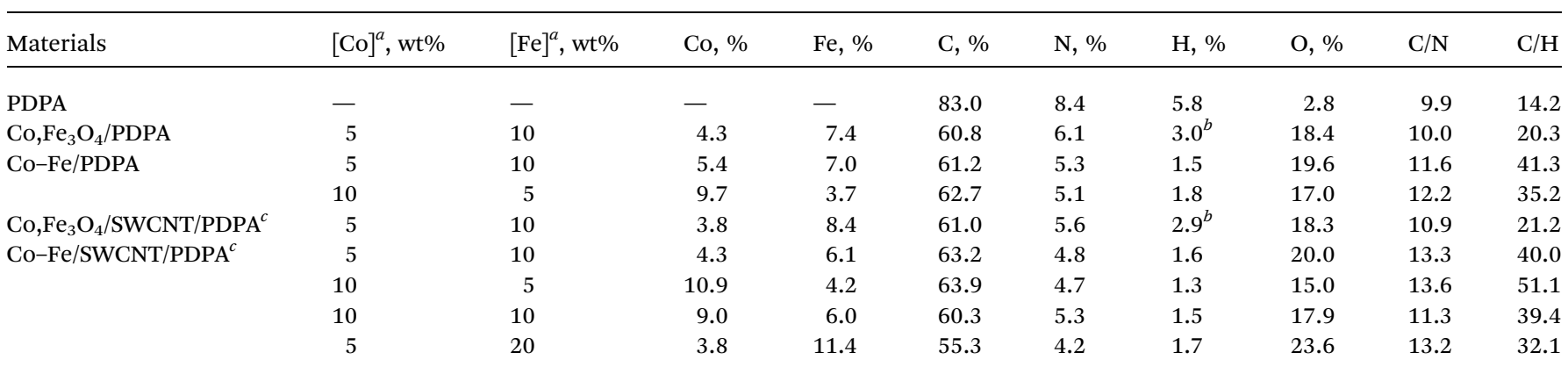

${ }^{a}[\mathrm{Co}]$ and $[\mathrm{Fe}] \mathrm{wt} \%$ at the loading. ${ }^{b} T=400{ }^{\circ} \mathrm{C}$, in other cases $T=600{ }^{\circ} \mathrm{C} .{ }^{c}[$ SWCNT $]=10 \mathrm{wt} \%$. 

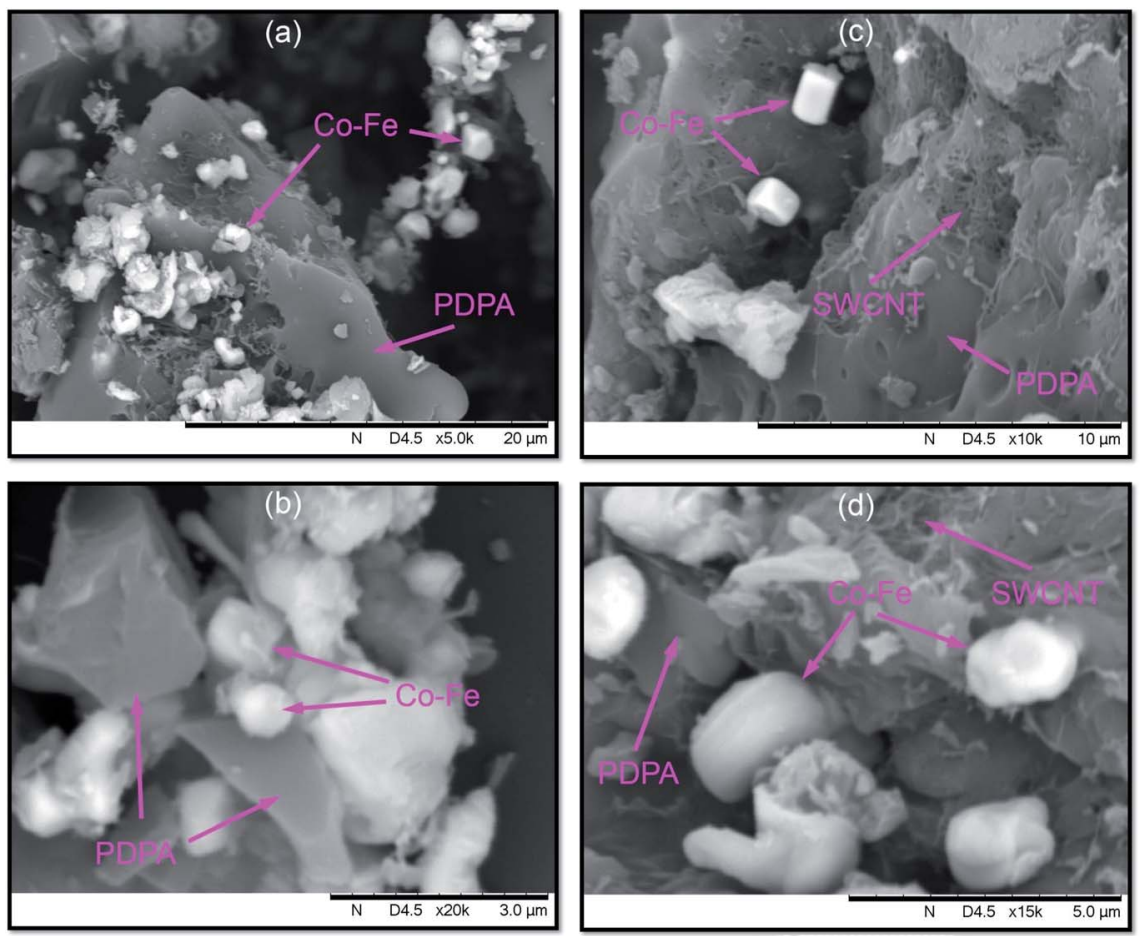

Fig. 4 SEM images of Co-Fe/PDPA ( $a$ and $b$ ) and Co-Fe/SWCNT/PDPA ( $c$ and $d$ ).

The impact of the synthesis conditions (sample temperature, heating time, concentration of Co and Fe, as well as SWCNT) on the yield and the phase composition of nanocomposites was studied. It was found that the yield of nanomaterials decreases with the increase in heating time and sample temperature; depending on the synthesis conditions, it is $58.3-76.0 \%(\mathrm{Co}-\mathrm{Fe} /$ PDPA) and 63.7-77.4\% (Co-Fe/SWCNT/PDPA) (see Table 2). According to ICP-AES data, the content of Co $=3.8-10.9 \mathrm{wt} \%$, and the content of $\mathrm{Fe}=3.7-11.4 \mathrm{wt} \%$ (see Table 4).

The study of the sample temperature effect on the phase composition of $\mathrm{Co}-\mathrm{Fe} / \mathrm{PPOA}$ and $\mathrm{Co}-\mathrm{Fe} / \mathrm{SWCNT} / \mathrm{PDPA}$ nanocomposites showed that the formation of bimetallic $\mathrm{Co}-\mathrm{Fe}$ particles occurs at temperatures above $400{ }^{\circ} \mathrm{C}$. At $T=450$ $500{ }^{\circ} \mathrm{C}$ and concentrations of $[\mathrm{Co}]=5 \mathrm{wt} \%$ and $[\mathrm{Fe}]=10 \mathrm{wt} \%$ at the loading, nanocomposites contain not only bimetallic $\mathrm{Co}-\mathrm{Fe}$ particles, but also $\beta$-Co particles with a cubic face-centered lattice, which is confirmed by the presence of reflections at diffraction angles $2 \theta=68.12^{\circ}, 80.94^{\circ}$, as well as $\mathrm{Fe}_{3} \mathrm{O}_{4}$ particles with reflection peaks in the range of $2 \theta=46.01^{\circ}, 54.08^{\circ}, 66.54^{\circ}$, $84.27^{\circ}, 90.82^{\circ}, 101.46^{\circ}$ (Fig. 3). In an inert atmosphere at $T=$ $600{ }^{\circ} \mathrm{C}$ under IR heating for $2-10 \mathrm{~min}$ at concentrations of [Co] $=5 \mathrm{wt} \%$ and $[\mathrm{Fe}]=10-20 \mathrm{wt} \%$ at the loading, only bimetallic $\mathrm{Co}-\mathrm{Fe}$ particles were registered. The increase in the concentration of iron to $22 \mathrm{wt} \%$ at the loading leads to the formation of $\mathrm{Fe}_{3} \mathrm{O}_{4}$ particles due to the excess of iron in relation to cobalt (Fig. 6a). On the contrary, at $[\mathrm{Co}]=10 \mathrm{wt} \%$ and $[\mathrm{Fe}]=5-10 \mathrm{wt} \%$, the diffraction patterns of nanocomposites show reflection peaks not only of bimetallic Co-Fe particles, but also of $\beta$-Co particles (Fig. 6b).
An interesting feature was observed when studying the effect of SWCNT concentration on the phase composition of $\mathrm{Co}-\mathrm{Fe} /$ SWCNT/PDPA nanocomposites. If the content of carbon nanotubes $[\mathrm{SWCNT}]=1-5 \mathrm{wt} \%$ relative to the polymer weight, the diffractograms of the nanocomposites contain not only reflection peaks of the Co-Fe particles, but also reflection peaks of the fcc phase of the solid solution based on the $\beta$-Co crystal lattice with the maximum intensity at [SWCNT] $=3 \mathrm{wt} \%$ (Fig. $6 \mathrm{c}$ ). The inset shows line diagrams for $\beta$-Co and Co-Fe. When the SWCNT content is increased up to $10 \mathrm{wt} \%$, only Co-Fe particles are registered, as in $\mathrm{Co}-\mathrm{Fe} / \mathrm{PDPA}$ nanocomposites obtained in the absence of SWCNT (Fig. 3f).

\subsection{Thermal properties of nanomaterials}

TGA and DSC methods were used to study thermal stability of the $\mathrm{Co}-\mathrm{Fe} / \mathrm{PDPA}$ and $\mathrm{Co}-\mathrm{Fe} / \mathrm{SWCNT} / \mathrm{PDPA}$ nanocomposites prepared at $600{ }^{\circ} \mathrm{C}$ for $10 \mathrm{~min}$ at $[\mathrm{Co}]=5 \mathrm{wt} \%$ and $[\mathrm{Fe}]=10 \mathrm{wt} \%$ at the loading. Fig. 7 shows the temperature dependence on the decrease in the weight of nanocomposites compared to PDPA at heating up to $1000{ }^{\circ} \mathrm{C}$ in the nitrogen flow and in air. Table 5 shows the main thermal characteristics of materials.

The Co-Fe/PDPA and Co-Fe/SWCNT/PDPA nanomaterials have high thermal stability. Approximately $9 \%$ weight loss occurs due to the presence of moisture in the nanocomposites, which is also confirmed by DSC data (Fig. 8). The DSC thermograms of the nanomaterials show an endothermic peak at $100{ }^{\circ} \mathrm{C}$. In the second heating this peak is absent.

After the moisture is removed, the weight of nanocomposites does not change until $350-380{ }^{\circ} \mathrm{C}$ in air. The processes of thermal oxidative destruction of $\mathrm{Co}-\mathrm{Fe} / \mathrm{PDPA}$ and $\mathrm{Co}-\mathrm{Fe} /$ 

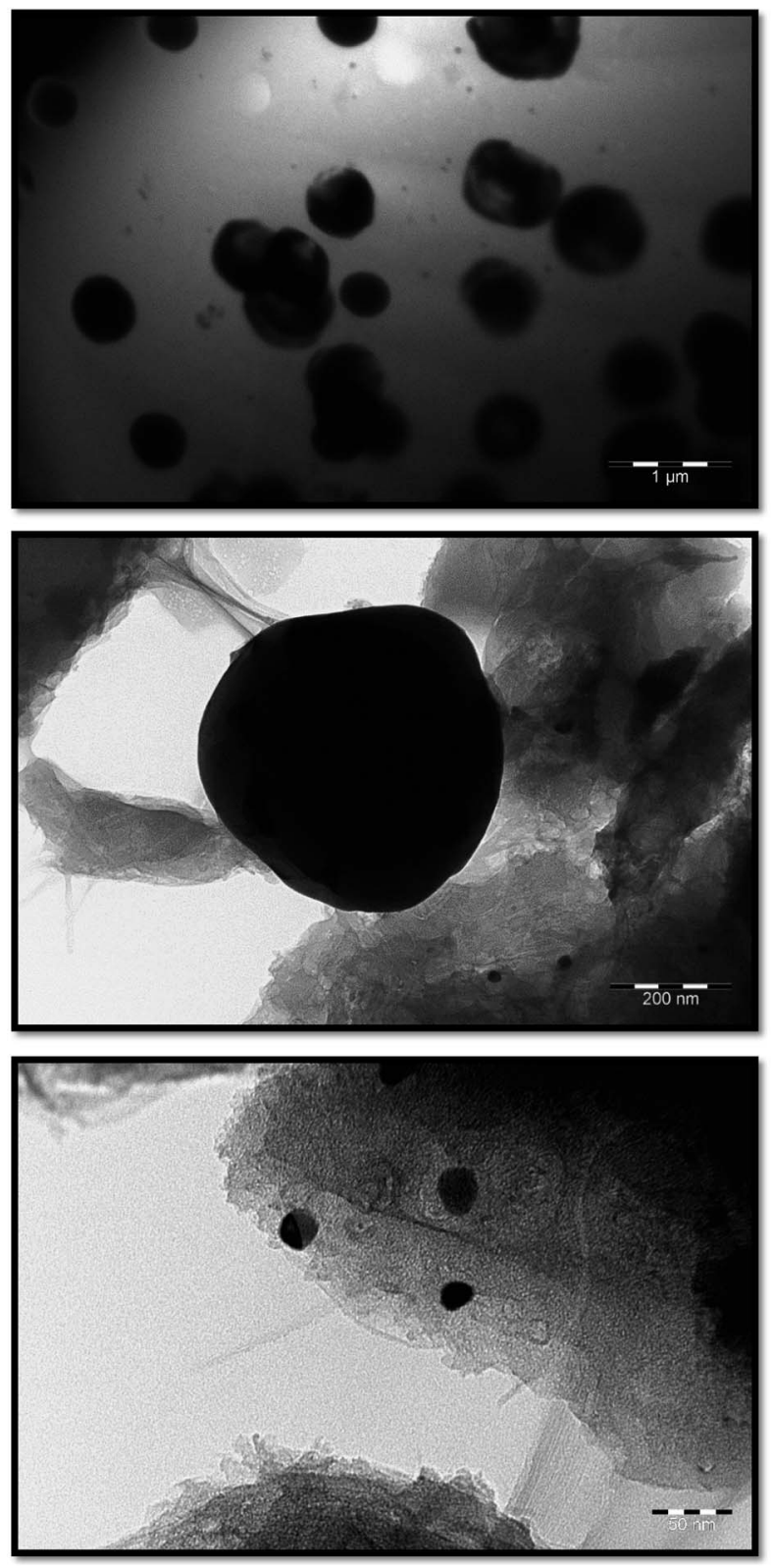

Fig. 5 TEM images of Co-Fe/SWCNT/PDPA.

SWCNT/PDPA nanocomposites begin at $400{ }^{\circ} \mathrm{C}$, in PDPA they begin at $470{ }^{\circ} \mathrm{C}$. A $50 \%$ weight loss in air is observed at $698{ }^{\circ} \mathrm{C}$ in PDPA. The Co-Fe/PDPA and Co-Fe/SWCNT/PDPA nanomaterials lose half of their initial weight in air at 660 and $580{ }^{\circ} \mathrm{C}$, respectively. The higher thermal stability of PDPA in air is associated with the fact that, as the temperature rises, in the neat polymer there is the process of oligomer polycondensation induced by atmospheric oxygen. ${ }^{64}$ At the same time, an increase in the polymerization degree of PDPA and a sharp decrease in the content of the crystalline low-molecular fraction are observed. For the Co-Fe/PDPA and Co-Fe/SWCNT/PDPA nanocomposites in air at $1000{ }^{\circ} \mathrm{C}$, the residue is 20 and $18 \%$, respectively. At the same time, according to ICP-AES data, the metal content in Co-Fe/PDPA is $5.4 \%$ Co and $7.0 \% \mathrm{Fe}$, which is
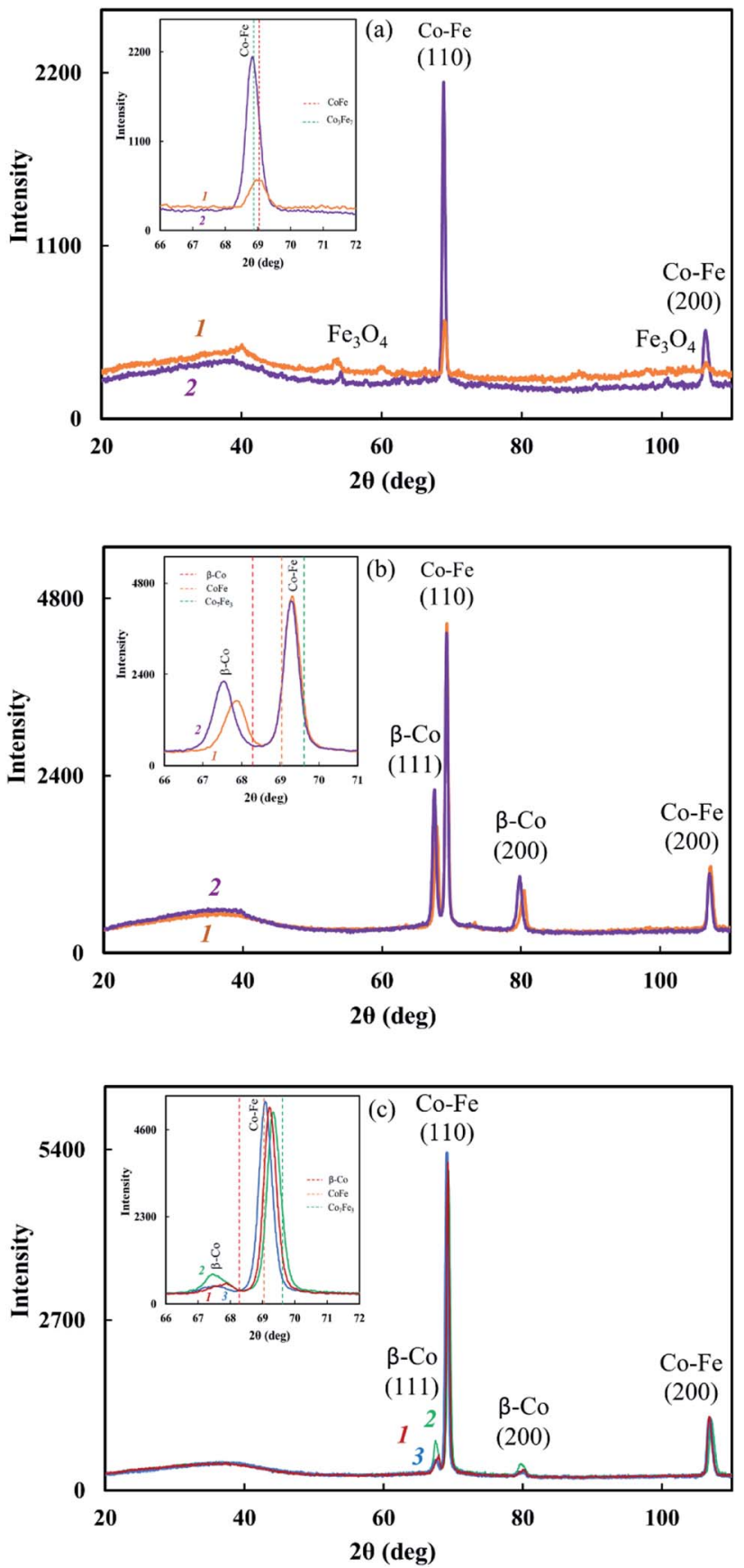

Fig. 6 XRD patterns of Co-Fe/PDPA ( $1 a$ and $1 b$ ) and Co-Fe/SWCNT/ PDPA (2a, 2b and $1 \mathrm{c}-3 \mathrm{c})$, prepared at $600{ }^{\circ} \mathrm{C},[\mathrm{Co}]=3$ (a), 5 (c), $10 \mathrm{wt} \%$ (b) and $[\mathrm{Fe}]=5$ (b), 10 (c), $22 \mathrm{wt} \%$ (a), [SWCNT] = 0 (1a and 1b), 1 (1c), 3 (2c), 5 (3c), 10 wt\% ( $2 a$ and $2 b$ ). The insets show line diagrams for $\beta$-Co and $\mathrm{Co}-\mathrm{Fe}$.

slightly higher than in Co-Fe/SWCNT/PDPA - 4.3\% Co and 6.1\% Fe (Table 4).

In an inert medium in neat PDPA in the temperature range of 300-400 ${ }^{\circ} \mathrm{C}$, the decomposition of diphenylamine oligomers ( $\sim 15 \%$ ) occurs, followed by some stabilization. PDPA loses half of its initial weight in an inert atmosphere at $880{ }^{\circ} \mathrm{C}$, whereas a gradual weight loss is observed in the Co-Fe/PDPA and Co-Fe/ 


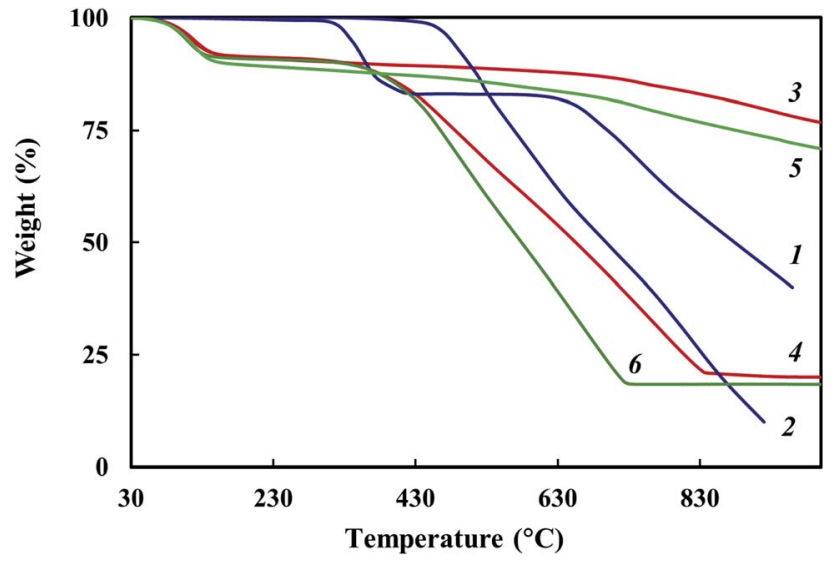

Fig. 7 TGA thermograms of PDPA (1 and 2), Co-Fe/PDPA (3 and 4) and Co-Fe/SWCNT/PDPA (5 and 6) at heating up to $1000{ }^{\circ} \mathrm{C}$ at the rate of $10{ }^{\circ} \mathrm{C} \mathrm{min}^{-1}$ in the nitrogen flow $(1,3$ and 5$)$ and in air $(2,4$ and 6).

Table 5 Thermal properties of materials

\begin{tabular}{llll}
\hline Materials & ${ }^{a} T_{5 \%},{ }^{\circ} \mathrm{C}$ & ${ }^{b} T_{50 \%},{ }^{\circ} \mathrm{C}$ & ${ }^{c}$ Residue, $\%$ \\
\hline PDPA & $490 / 340$ & $698 / 880$ & $0 / 40$ \\
Co-Fe/PDPA & $116 / 119$ & $660 />1000$ & $20 / 77$ \\
Co-Fe/SWCNT/ & $109 / 112$ & $580 />1000$ & $18 / 71$
\end{tabular}

PDPA $^{d}$

${ }^{a} T_{5 \%}-5 \%$ weight losses (air/argon). ${ }^{b} T_{50 \%}-50 \%$ weight losses (air/ argon). ${ }^{c}$ Residue at $1000{ }^{\circ} \mathrm{C}$ (air/argon). ${ }^{d}$ [SWCNT] $=10 \mathrm{wt} \%$.

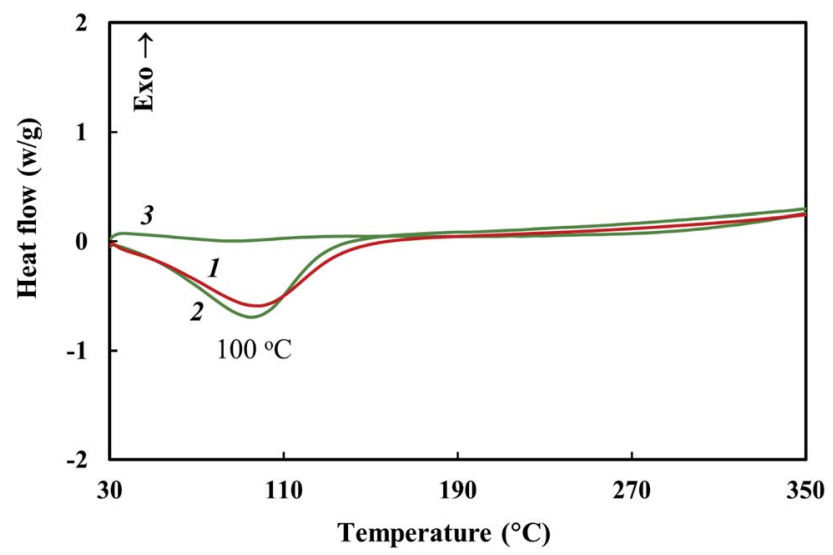

Fig. 8 DSC thermograms of Co-Fe/PDPA (1) and Co-Fe/SWCNT/ PDPA (2 and 3) at heating in the nitrogen flow to $350{ }^{\circ} \mathrm{C}$ at the rate of $10^{\circ} \mathrm{C} \min ^{-1}$ ( 1 and 2 - first heating, 3 - second heating).

SWCNT/PDPA nanocomposites; at $1000{ }^{\circ} \mathrm{C}$, the residue is 77 and $71 \%$, respectively.

\subsection{Electrical properties of nanomaterials}

Frequency dependence on $a c$ conductivity of the Co-Fe/PDPA and $\mathrm{Co}-\mathrm{Fe} / \mathrm{SWCNT} / \mathrm{PDPAC}$ nanocomposites obtained at $600{ }^{\circ} \mathrm{C}$ during $10 \mathrm{~min}$ at $[\mathrm{Co}]=5 \mathrm{wt} \%$ and $[\mathrm{Fe}]=10 \mathrm{wt} \%$ at the loading compared to neat PDPA and PDPA treated at $600{ }^{\circ} \mathrm{C}$ was studied (Fig. 9). The composition of the metallic phase corresponds to the Co-Fe phase (Fig. 3f).

According to, ${ }^{77-79}$ the frequency dependence on the conductivity $\left(\sigma_{\mathrm{ac}}\right)$ is described by equation:

$$
\sigma_{\mathrm{ac}}=\sigma_{\mathrm{dc}}+A \omega^{n},
$$

where $\omega=2 \pi f$ is the angular frequency, $\sigma_{\mathrm{dc}}$ - the frequency independent (dc) part of conductivity, $n$ - the exponential parameter $(0 \leq n \leq 1), A-$ the thermally activated quantity. $A$ and $n$ depend on the temperature and the volume fraction of the conducting component.

Table 6 shows the values of conductivity $\left(\sigma_{\mathrm{ac}}\right)$ of materials at $25 \mathrm{~Hz}$ and $1 \mathrm{MHz}$. Neat PDPA demonstrates a low conductivity value. $\sigma_{\mathrm{dc}}$ for PDFA is $5.2 \times 10^{-11} \mathrm{~S} \mathrm{~cm}^{-1}$. As frequency grows, the electrical conductivity $\sigma_{\mathrm{ac}}$ of the polymer increases linearly from $6.3 \times 10^{-1} \mathrm{~S} \mathrm{~cm}^{-1}$ to $3.1 \times 10^{-6} \mathrm{~S} \mathrm{~cm}^{-1}$. The approximation by the equation of the frequency dependence on the electrical conductivity gives the value of $n=0.99$, which indicates the hopping conductivity mechanism $(0 \leq n \leq 1)$, typical of most conductive polymers. ${ }^{77-80}$

Under heat treatment of PDPA at $600{ }^{\circ} \mathrm{C}$, electrical conductivity $\sigma_{\mathrm{ac}}$ increases significantly to $(7.3-8.5) \times 10^{-5} \mathrm{~S} \mathrm{~cm}^{-1}(f=$ $25-10^{6} \mathrm{~Hz}$ ), while $\sigma_{\mathrm{dc}}=7 \times 10^{-5} \mathrm{~S} \mathrm{~cm}^{-1}, n=0.98$. Thus, the mechanism of electrical conductivity has not changed. The observed increase in the conductivity $\sigma_{\mathrm{dc}}$ is caused by the increase in the size of the conducting regions, which are areas of continuous conjugation resulting from dehydrogenation reactions with the formation of $\mathrm{C}=\mathrm{N}$ double bonds and polycondensation of DPA oligomers. As shown above, IR treated at $600{ }^{\circ} \mathrm{C}$, PDPA is amorphous (Fig. 3a). Thus, heat treatment of PDFA leads to amorphization of the polymer due to phase and morphological transformations. The formation of an extended conjugated system during heat treatment causes an increase in the degree of percolation and leads to an increase in electrical conductivity.

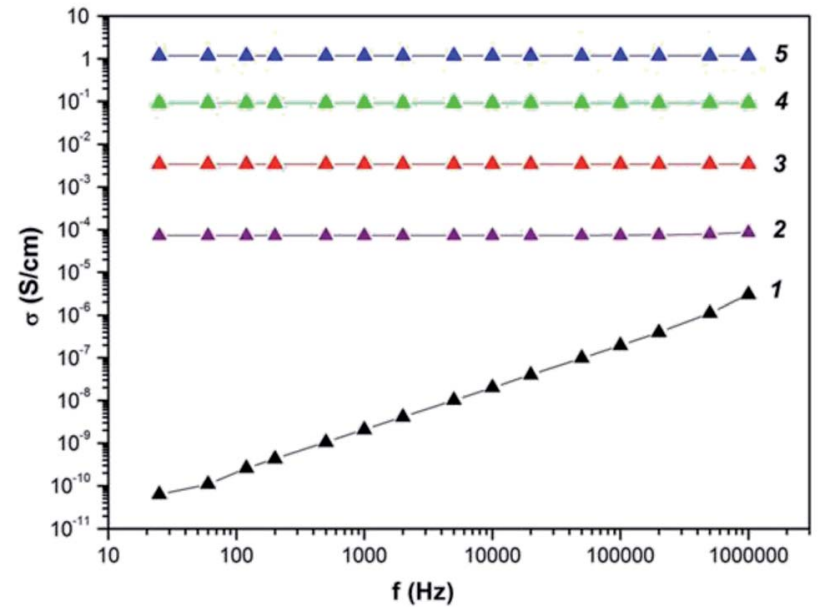

Fig. 9 Frequency dependence on the conductivity for PDPA (1) and PDPA at $600{ }^{\circ} \mathrm{C}$ (2), Co-Fe/PDPA (3) and Co-Fe/SWCNT/PDPA, prepared at $[S W C N T]=3(4)$ and $10 \mathrm{wt} \%$ (5). 
Table 6 The conductivity values of materials

\begin{tabular}{|c|c|c|c|c|c|}
\hline Materials & & ${ }^{a} \sigma_{\mathrm{ac}}, \mathrm{S} \mathrm{cm}^{-1}$ & & $\sigma_{\mathrm{dc}}, \mathrm{S} \mathrm{cm}^{-1}$ & $n$ \\
\hline \multirow[t]{2}{*}{ PDPA } & Neat & $6.3 \times 10^{-11}$ & $3.1 \times 10^{-6}$ & $5.2 \times 10^{-11}$ & 0.99 \\
\hline & Heated & $7.3 \times 10^{-5}$ & $8.5 \times 10^{-5}$ & $7.0 \times 10^{-5}$ & 0.98 \\
\hline \multirow{2}{*}{$\begin{array}{l}\mathrm{Co}-\mathrm{Fe} / \mathrm{PDPA} \\
{ }^{b} \mathrm{Co}-\mathrm{Fe} / \mathrm{SWCNT} / \mathrm{PDPA}\end{array}$} & 3 & $9.2 \times 10^{-2}$ & $9.3 \times 10^{-2}$ & $8.5 \times 10^{-2}$ & 0.001 \\
\hline & 10 & 1.2 & 1.3 & $9.7 \times 10^{-1}$ & 0.001 \\
\hline
\end{tabular}

The presence of bimetallic Co-Fe particles in the matrix of heat-treated PDPA increases the degree of percolation even more, because metals have a high inherent electrical conductivity. As a result, $\sigma_{\mathrm{ac}}$ of the Co-Fe/PDPA nanocomposite increases to $3.4 \times 10^{-3} \mathrm{~S} \mathrm{~cm}^{-1}$ and demonstrates weak dependency on frequency $(n=0.002)$. Apparently, the presence of metal particles helps the formation of large conducting regions, which leads to exceeding the percolation threshold. In this case, no change in the conductivity of the Co-Fe/PDPA nanocomposite in the high-frequency region is observed.

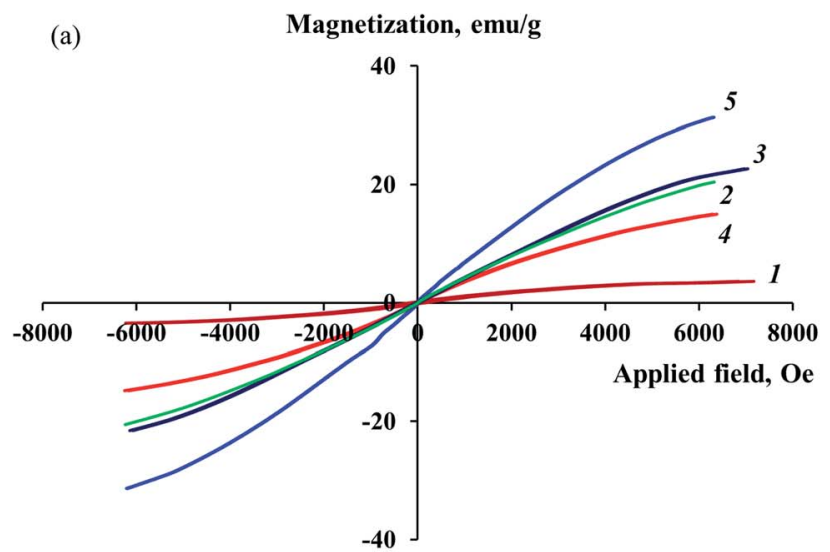

(b)

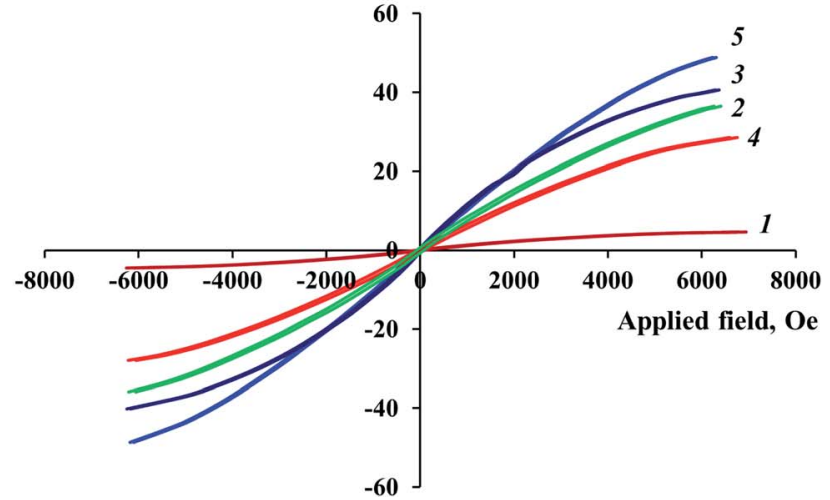

Fig. 10 Magnetization of $\mathrm{Co}, \mathrm{Fe}_{3} \mathrm{O}_{4} / \mathrm{PDPA}$ (1a), $\mathrm{Co}, \mathrm{Fe}_{3} \mathrm{O}_{4} / \mathrm{SWCNT} /$ PDPA (1b), Co-Fe/PDPA (2a-5a) and Co-Fe/SWCNT/PDPA (2b-5b), prepared at $400(1)$ and $600{ }^{\circ} \mathrm{C}(2-5),[\mathrm{Co}]=5(1,2,3), 10 \mathrm{wt} \%(4,5)$ and $[\mathrm{Fe}]=5(4), 10(1,2,5), 20 \mathrm{wt} \%$ (3), [SWCNT] $=10 \mathrm{wt} \%$ (b), as a function of applied magnetic field at room temperature.
When metal particles and SWCNT are simultaneously introduced into the structure of nanocomposites, an even greater increase in electrical conductivity is observed. For a nanocomposite containing $3 \mathrm{wt} \%$ of SWCNT, $\sigma_{\mathrm{ac}}=(9.2-9.3) \times$ $10^{-2} \mathrm{~S} \mathrm{~cm}^{-1}$ in the frequency range $25-10^{6} \mathrm{~Hz}(n=0.001)$, and for a nanocomposite containing $10 \mathrm{wt} \%$ of SWCNT, $\sigma_{\mathrm{ac}}=1.2-$ $1.3 \mathrm{~S} \mathrm{~cm}^{-1}(n=0.001)$. Just as for metal-polymer nanocomposites, a very weak frequency dependence on the conductivity is observed in Co-Fe/SWCNT/PDPA, which indicates that the percolation threshold is exceeded and a common conducting region is formed. An increase in the SWCNT content provides a decrease in resistance due to an increase in the number of conduction channels.

\subsection{Magnetic properties of nanomaterials}

The study of magnetic properties at room temperature showed that the obtained Co-Fe/PDPA and Co-Fe/SWCNT/PDPA nanocomposites demonstrate a hysteresis character of remagnetization. The magnetization dependency on the value of the applied magnetic field is shown in Fig. 10. Table 7 shows the values of the main magnetic properties of nanocomposites. As seen in Fig. 10, the saturation magnetization of nanomaterials grows with the increase in the concentration of both cobalt and iron, and reaches $M_{\mathrm{S}}=14.99-31.32 \mathrm{emu} \mathrm{g}^{-1}$ (Co-Fe/PDPA) and $M_{\mathrm{S}}=29.48-48.84 \mathrm{emu} \mathrm{g}^{-1}$ (Co-Fe/SWCNT/PDPA) at $H=7000$ Oe, depending on the synthesis conditions. The values of the coercive force $H_{\mathrm{C}}$ and residual magnetization $M_{\mathrm{R}}$ are nonzero for the studied Co-Fe/PDPA and Co-Fe/SWCNT/PDPA nanomaterials: $M_{\mathrm{R}}$ is $0.06-0.18 \mathrm{emu} \mathrm{g}^{-1}$ and $0.39-0.85 \mathrm{emu} \mathrm{g}^{-1}$, and $H_{\mathrm{C}}$ reaches 5-42 Oe and 27-80 Oe, respectively. This indicates the contribution of large (400-800 $\mathrm{nm}$ for Co-Fe/PDPA and 400$1100 \mathrm{~nm}$ for Co-Fe/SWCNT/PDPA, according to TEM data) ferromagnetic particles to the magnetization processes. Small nanoparticles formed during the synthesis of nanocomposites (8-30 nm and 23-50 nm for Co-Fe/PDPA and Co-Fe/SWCNT/ PDPA, respectively) have a superparamagnetic nature. The $M_{\mathrm{R}} /$ $M_{\mathrm{S}}$ ratio is 0.003-0.011 for Co-Fe/PDPA and 0.011-0.018 for CoFe/SWCNT/PDPA, which indicates the formation of both superparamagnetic and ferromagnetic particles. Higher values of the coercive force $H_{\mathrm{C}}$ and residual magnetization $M_{\mathrm{R}}$ in the Co-Fe/SWCNT/PDPA nanocomposites seem to be associated with the formation of larger Co-Fe particles. Nanocomposites obtained at $400{ }^{\circ} \mathrm{C}$ are characterized by low saturation magnetization $M_{\mathrm{S}}$ values and high coercive force (curves 1 in Fig. 10). 
Table 7 Magnetic properties of nanomaterials

\begin{tabular}{|c|c|c|c|c|c|c|c|c|c|}
\hline Nanomaterials & {$[\mathrm{Co}]^{a}, \mathrm{wt} \%$} & {$[\mathrm{Fe}]^{a}, \mathrm{wt} \%$} & $\mathrm{Co}^{b}, \%$ & $\mathrm{Fe}^{b}, \%$ & Co and Fe phase composition & $H_{\mathrm{C}}$, Oe & $M_{\mathrm{S}}, \mathrm{emu} \mathrm{g}^{-1}$ & $M_{\mathrm{R}}, \mathrm{emu} \mathrm{g}^{-1}$ & $M_{\mathrm{R}} / M_{\mathrm{S}}$ \\
\hline $\mathrm{Co}, \mathrm{Fe}_{3} \mathrm{O}_{4} / \mathrm{PDPA}^{c}$ & 5 & 10 & 4.3 & 7.4 & $\alpha-\mathrm{Co}, \beta-\mathrm{Co}, \mathrm{Fe}_{3} \mathrm{O}_{4}$ & 156 & 3.67 & 0.18 & 0.049 \\
\hline \multirow[t]{3}{*}{$\mathrm{Co}-\mathrm{Fe} / \mathrm{PDPA}$} & 5 & 10 & 5.4 & 7.0 & $\mathrm{Co}-\mathrm{Fe}$ & 5 & 20.43 & 0.06 & 0.003 \\
\hline & 10 & 5 & 9.7 & 3.7 & $\mathrm{Co}-\mathrm{Fe}, \beta-\mathrm{Co}$ & 42 & 14.99 & 0.17 & 0.011 \\
\hline & 10 & 10 & 9.2 & 7.5 & $\mathrm{Co}-\mathrm{Fe}, \beta-\mathrm{Co}$ & 11 & 31.32 & 0.18 & 0.006 \\
\hline $\mathrm{Co}, \mathrm{Fe}_{3} \mathrm{O}_{4} / \mathrm{SWCNT} / \mathrm{PDPA}^{c}$ & 5 & 10 & 3.8 & 8.4 & $\alpha-\mathrm{Co}, \beta-\mathrm{Co}, \mathrm{Fe}_{3} \mathrm{O}_{4}$ & 80 & 4.63 & 0.14 & 0.030 \\
\hline & 10 & 5 & 10.9 & 4.2 & Co-Fe, $\beta$-Co & 80 & 29.48 & 0.55 & 0.018 \\
\hline & 10 & 10 & 9.0 & 6.0 & Co-Fe, $\beta$-Co & 27 & 48.84 & 0.57 & 0.011 \\
\hline
\end{tabular}

${ }^{a}[\mathrm{Co}]$ and [Fe] wt $\%$ at the loading. ${ }^{b}$ According to ICP-AES data. ${ }^{c} T=400{ }^{\circ} \mathrm{C}$, in other cases $T=600{ }^{\circ} \mathrm{C}$. $[\mathrm{SWCNT}]=10 \mathrm{wt} \% . H_{\mathrm{C}}-\mathrm{coercive}$ force, $M_{\mathrm{S}}-$ saturation magnetization, $M_{\mathrm{R}}$ - residual magnetization.

This is due to the fact that at temperatures below $450{ }^{\circ} \mathrm{C}, \mathrm{Co}-\mathrm{Fe}$ particles have not formed. As shown above, nanomaterials containing $\alpha$-Co, $\beta$-Co and $\mathrm{Fe}_{3} \mathrm{O}_{4}$ particles are formed at $400{ }^{\circ} \mathrm{C}$.

Thus, the magnetic behavior of the studied nanocomposites is determined by the presence of small superparamagnetic and large ferromagnetic particles in their structure.

\section{Conclusions}

The one-stage method for the formation of hybrid electromagnetic nanomaterials based on PDPA and Co-Fe particles in the absence and presence of SWCNT under IR heating conditions makes it possible, without subjecting the polymer matrix to degradation, to obtain magnetic bimetallic particles directly during the synthesis of nanocomposites, and thereby to expand the range of magnetic nanoparticles and to obtain novel magnetic and electrically conductive nanomaterials. The originality of the proposed approach is determined by the fact that the $\mathrm{Co}-\mathrm{Fe} / \mathrm{PDPA}$ and $\mathrm{Co}-\mathrm{Fe} / \mathrm{SWCNT} / \mathrm{PDPA}$ nanomaterials are formed in situ with simultaneous reactions of polycondensation of DPA oligomers, dehydrogenation of phenyleneamine units of the polymer matrix and the reduction of metals by evolved hydrogen under IR heating. The saturation magnetization of nanomaterials increases with an increase in the concentration of both cobalt and iron, and at $H=7000$ Oe it is $M_{\mathrm{S}}=14.99-$ $31.32 \mathrm{emu} \mathrm{g}^{-1}$ (Co-Fe/PDPA) and $M_{\mathrm{S}}=29.48-48.84 \mathrm{emu} \mathrm{g}^{-1}$ (Co-Fe/SWCNT/PDPA). In this case, the $M_{\mathrm{R}} / M_{\mathrm{S}}$ ratio is $0.003-$ 0.011 (Co-Fe/PDPA) and 0.011-0.018 (Co-Fe/SWCNT/PDPA). The formation of a mixture of superparamagnetic and ferromagnetic particles is associated with the fact that both small and large Co-Fe particles are formed during the synthesis of nanomaterials. Nanocomposites are characterized by a weak frequency dependence on electrical conductivity. An increase in the electrical conductivity of nanomaterials occurs due to the presence of both bimetallic Co-Fe particles and SWCNT and reaches $3.5 \times 10^{-3} \mathrm{~S} \mathrm{~cm}^{-1}(\mathrm{Co}-\mathrm{Fe} / \mathrm{PDPA})$ and $1.3 \mathrm{~S} \mathrm{~cm}^{-1}(\mathrm{Co}-$ $\mathrm{Fe} / \mathrm{SWCNT} / \mathrm{PDPA})$. In an inert medium, a gradual weight loss is observed in nanocomposites, and at $1000{ }^{\circ} \mathrm{C}$ the residue is $71-$ $77 \%$. Prepared electromagnetic nanomaterials have potential applications in the manufacture of electrochemical devices, such as sensors and nanoprobes, rechargeable batteries, supercapacitors, as well as at fabrication of electromagnetic shielding and corrosion resistant coatings, etc.

\section{Author contributions}

Conceptualization, investigation and writing - original draft, S. Z. O.; supervision, writing - review \& editing, G. P. K.; investigation, M. N. E, A. A. V., D. G. M., V. A. P., P. A. Ch., G. V. P.

\section{Funding}

This research received no external funding.

\section{Conflicts of interest}

The authors declare no conflicts of interest.

\section{Acknowledgements}

This work was carried out within the State Program of TIPS RAS. This work was performed using the equipment of the Shared Research Center «Analytical center of deep oil processing and petrochemistry of TIPS RAS.

\section{References}

1 F. S. A. Khan, N. M. Mubarak, Y. H. Tan, R. R. Karri, M. Khalid, R. Walvekar, E. Ch. Abdullah, Sh. A. Mazari and S. Nizamuddin, Environ. Sci. Pollut. Res., 2020, 27, 4352643541.

2 L. Zhang, W. Y. Du, A. Nautiyal, Z. Liu and X. Y. Zhang, Sci. China Mater., 2018, 61, 303-352.

3 D. W. Jiang, V. Murugadoss, Y. Wang, J. Lin, T. Ding, Z. C. Wang, Q. Shao, C. Wang, H. Liu and N. Lu, Polym. Rev., 2019, 59, 280-337.

4 I. Sapurina and J. Stejskal, Polym. Int., 2008, 57, 1295-1325.

5 I. Yu. Sapurina and J. Stejskal, Russ. Chem. Rev., 2010, 79, $1123-1143$. 
6 E. Song and J. W. Choi, Nanomaterials, 2013, 3, 498-523.

7 E. Song and J. Choi, Nanomaterials, 2013, 3, 498-523.

8 S. Ghosh, T. Maiyalagan and R. N. Basu, Nanoscale, 2016, 8, 6921-6947.

9 M. Sh. Zoromba, A. A. Alshehri, A. F. Al-Hossainy and M. H. Abdel-Aziz, Opt. Mater., 2021, 111, 110621.

10 M. Sh. Zoromba, M. H. Abdel-Aziz, M. Bassyouni, A. Attar and A. F. Al-Hossainy, J. Mol. Struct., 2021, 1225, 129131.

11 N. Almutlaq, A. F. Al-Hossainy and M. Sh. Zoromba, J. Mol. Struct., 2021, 1227, 129712.

12 E. Tomsík, O. Kohut, I. Ivanko, M. Pekarek, I. Bieloshapka and P. Dallas, J. Phys. Chem., 2018, 122, 8022-8030.

13 A. F. Al-Hossainy and M. Sh. Zoromba, Appl. Phys. A, 2021, 127, 278.

14 A. Bourezgui, A. F. Al-Hossainy, I. H. El. Azab, F. Alresheedi, S. A. Mahmoud, M. Bassyouni, M. H. Abdel-Aziz and M. Sh. Zoromba, J. Mater. Sci.: Mater. Electron., 2021, 32, 5489-5503.

15 Y. Wang, H. D. Tran, L. Liao, X. Duan and R. B. Kaner, J. Am. Chem. Soc., 2010, 132, 10365-10373.

16 M. Sh Zoromba, A. F. Al-Hossainy, M. Rzaigui, A. Abdelkader, F. Alresheedi, I. H. El. Azab and F. M. Eissa, Opt. Mater., 2021, 112, 110758.

17 A. Ibrahim, M. H. Abdel-Aziz, M. S. Zorombam and A. F. AlHossainy, Synth. Met., 2018, 238, 1-13.

18 H. Lin, Q. Huang, J. Wang, J. Jiang, F. Liu, Y. Chen, C. Wang, D. Lu and S. Han, Electrochim. Acta, 2016, 191, 444-451.

19 Z. He, Y. Fang, X. Wang and H. Pang, Synth. Met., 2011, 161, 420-425.

20 R. B. Yang, P. M. Reddy, C. J. Chang, P. A. Chen, J. K. Chen and C. C. Chang, Chem. Eng. J., 2016, 285, 497-507.

21 G. Ren, Y. Li, Z. Guo, G. Xiao, Y. Zhu, L. Dai and L. Jiang, Nano Res., 2015, 8, 3461-3471.

22 P. Xiong, H. Huang and X. Wang, J. Power Sources, 2014, 245, 937-946.

23 K. Zhang, X. Zhang, X. Zhao, X. Gai, W. An, G. Fang, A. Zhang and X. Chen, J. Mater. Sci.: Mater. Electron., 2019, 30, 1733317341.

24 M. Alsultan, J. Choi, P. Wagner and G. F. Swiegers, ChemCatChem, 2020, 12, 1580-1584.

25 L. Gao, R. Yue, J. Xu and Z. Liu, Int. J. Electrochem. Sci., 2018, 13, 6791-6802.

26 N. H. N. Azman, M. S. Mamat, H. N. Lim and Y. Sulaiman, J. Mater. Sci.: Mater. Electron., 2018, 29, 6916-6923.

27 S. F. Hojati, A. Amiri, N. MoeiniEghbali and S. Mohamadi, Appl. Organomet. Chem., 2018, 32, E423.

28 J. Dalal, S. Lather, A. Gupta, R. Tripathi, A. S. Maan, K. Singh and A. Ohlan, Adv. Mater. Technol., 2019, 4, 1900023.

29 Y. J. Zhang, Y. W. Lin, C. C. Chang and T. M. Wu, Synth. Met., 2011, 161, 937-942.

30 T. Chen, J. Qiu, K. Zhu, Y. Che, Y. Zhang, J. Zhang, H. Li, F. Wang and Z. Wang, J. Mater. Sci.: Mater. Electron., 2014, 25, 3664-3673.

31 T. M. Wu, S. J. Yen, E. C. Chen and R. K. Chiang, J. Polym. Sci., Part B: Polym. Phys., 2008, 46, 727-733.

32 S. Radhakrishnan, K. Krishnamoorthy, C. Sekar, J. Wilson and S. J. Kim, Chem. Eng. J., 2015, 259, 594-602.
33 L. Kong, X. Lu and W. Zhang, J. Solid State Chem., 2008, 181, 628-636.

34 M. S. Cao, J. Yang, W. L. Song, D. Q. Zhang, B. Wen, H. B. Jin, Z. L. Hou and J. Yuan, ACS Appl. Mater. Interfaces, 2012, 4, 6949-6956.

35 H. Zhou, X. Wang, K. Yu, C. Zhang, H. Li and Z. Du, Integr. Ferroelectr., 2014, 154, 159-165.

36 P. Liu, Y. Huang and X. Zhang, J. Alloys Compd., 2014, 596, 25-31.

37 P. Liu, Y. Huang, L. Wang and W. Zhang, Synth. Met., 2013, 177, 89-93.

38 A. P. Singh, M. Mishra, P. Sambyal, B. K. Gupta, B. P. Singh, A. Chandra and S. K. Dhawan, J. Mater. Chem. A, 2014, 2, 3581-3593.

39 Y. Liu, J. Li, F. Li, W. Li, H. Yang, X. Zhang, Y. Liu and J. Ma, J. Mater. Chem. A, 2016, 4, 4472-4478.

40 S. Giri, D. Ghosh and C. K. Das, J. Electroanal. Chem., 2013, 697, 32-45.

41 C. Zhao, Y. Jin, X. Du and W. Du, J. Power Sources, 2018, 399, 337-342.

42 S. Wang, H. Bao, P. Yang and G. Chen, Anal. Chim. Acta, 2008, 612, 182-189.

43 A. Zhu, P. Shi, S. Sun and M. Rui, Prog. Org. Coat., 2019, 133, 117-124.

44 Y. Ma, Y. Zhou, Y. Sun, H. Chen, Z. Xiong, X. Li, L. Shen and Y. Liu, J. Alloys Compd., 2019, 796, 120-130.

45 J. Luo, Y. Xu, W. Yao, C. Jiang and J. Xu, Compos. Sci. Technol., 2015, 117, 315-321.

46 A. Moyseowicz, A. Sliwak, E. Miniach and G. Gryglewicz, Composites, Part B, 2017, 109, 23-29.

47 X. Lu, H. Mao, D. Chao, W. Zhang and Y. Wei, J. Solid State Chem., 2006, 179, 2609-2615.

48 S. Zh. Ozkan, G. P. Karpacheva, P. A. Chernavskii, E. L. Dzidziguri, G. N. Bondarenko and G. V. Pankina, Polymers, 2018, 10, 544.

49 S. Zh. Ozkan, A. I. Kostev, G. P. Karpacheva, P. A. Chernavskii, A. A. Vasilev and D. G. Muratov, Polymers, 2020, 12, 1568.

50 S. Zh. Ozkan, A. I. Kostev and G. P. Karpacheva, Polym. Bull., 2021, DOI: 10.1007/s00289-021-03558-4.

51 S. Koutsopoulos, R. Barfod, K. M. Eriksen and R. Fehrmann, J. Alloys Compd., 2017, 725, 1210-1216.

52 K. Zehani, R. Bez, A. Boutahar, E. K. Hlil, H. Lassri, J. Moscovici, N. Mliki and L. Bessais, J. Alloys Compd., 2014, 591, 58-64.

53 E. V. Yakushko, D. G. Muratov, L. V. Kozhitov, E. Y. Korovin, A. A. Lomov and A. V. Popkova, Russ. Phys. J., 2021, 63, 22262235.

54 D. G. Muratov, A. A. Vasilev, M. N. Efimov, G. P. Karpacheva, E. L. Dzidziguri and P. A. Chernavskiy, Inorg. Mater., 2019, 10, 666-672.

55 S. Zh. Ozkan, G. P. Karpacheva, P. A. Chernavskii, E. L. Dzidziguri, G. N. Bondarenko, M. N. Efimov and G. V. Pankina, Polym. Bull., 2017, 74, 3043-3060.

56 S. Zh. Ozkan, G. P. Karpacheva, E. L. Dzidziguri, P. A. Chernavskii, G. N. Bondarenko and G. V. Pankina, J. Res. Updates Polym. Sci., 2017, 5, 137-148. 
57 S. Zh. Ozkan, G. P. Karpacheva, E. L. Dzidziguri, M. N. Efimov, G. N. Bondarenko, G. A. Shandryuk, P. A. Chernavskii and G. V. Pankina, J. Polym. Res., 2019, 26, 176.

58 S. Zh. Ozkan, E. L. Dzidziguri, G. P. Karpacheva, P. A. Chernavskii, M. N. Efimov and G. N. Bondarenko, Russ. Chem. Bull., 2015, 64, 196-201.

59 S. Zh. Ozkan, E. L. Dzidziguri, P. A. Chernavskii, G. P. Karpacheva, M. N. Efimov and G. N. Bondarenko, Nanotechnol. Russ., 2013, 8, 452-460.

60 G. P. Karpacheva, S. Zh. Ozkan, E. L. Dzidziguri, P. A. Chernavskii, I. S. Eremeev, M. N. Efimov, M. I. Ivantsov and G. N. Bondarenko, Eur. Chem. Bull., 2015, 4, 135-141.

$61 \mathrm{H}$. Yang and A. J. Bard, J. Electroanal. Chem. Interfacial Electrochem., 1991, 306, 87-109.

62 A. V. Orlov, S. Zh. Ozkan, G. N. Bondarenko and G. P. Karpacheva, Polym. Sci., Ser. B, 2006, 48, 5-10.

63 A. V. Orlov, S. Zh. Ozkan and G. P. Karpacheva, Polym. Sci., Ser. $B, 2006,48,11-17$.

64 S. Zh. Ozkan, G. P. Karpacheva, A. V. Orlov and M. A. Dzyubina, Polym. Sci., Ser. B, 2007, 49, 36-41.

65 M. H. Kim, D. H. Bae, H. J. Choi and Y. Sen, Polymer, 2017, 119, 40-49.

66 A. I. Gopalan, K.-P. Lee, K. M. Manesh and P. Santhosh, J. Membr. Sci., 2008, 318, 422-428.

67 M. Baibarac, I. Baltog, S. Lefrant and P. Gomez-Romero, Mater. Sci. Eng., B, 2011, 176, 110-120.
68 T. Permpool, A. Sirivat and D. Aussawasathien, Sens. Actuators, B, 2015, 220, 91-100.

69 F. A. Obreskov, A. F. Shestakov, S. G. Vasil'ev, K. J. Stevenson and P. A. Troshin, J. Mater. Chem. A, 2021, 9, 2864.

70 H. Jang, S. Kwon, J. Lee and H. Choi, Polymer, 2019, 182, 121851.

71 S. Zh. Ozkan and G. P. Karpacheva, RU patent for the invention, Utility patents, No 2724251 C1 from 22. 06. 2020.

72 M. N. Efimov, A. A. Vasilev, D. G. Muratov, A. E. Baranchikov and G. P. Karpacheva, J. Environ. Chem. Eng., 2019, 7, 103514.

73 M. N. Efimov, V. E. Sosenkin, Y. M. Volfkovich, A. A. Vasilev, D. G. Muratov, S. A. Baskakov, O. N. Efimov and G. P. Karpacheva, Electrochem. Commun., 2018, 96, 98-102.

$74 \mathrm{~S}$. Zh. Ozkan and G. P. Karpacheva, RU patent for the invention, Utility patents, No 2737184 C1 from 25.11.2020.

75 P. A. Chernavskii, G. V. Pankina and V. V. Lunin, Russ. Chem. Rev., 2011, 80, 579-604.

76 PDF-2, The International Centre for Diffraction Data, http:// www.icdd.com/index.php/pdf-2/, 2021 [accessed 08 April 2021].

77 A. K. Jonscher, Nature, 1977, 267, 673-679.

78 J. C. Dyre and T. B. Schrøder, Rev. Mod. Phys., 1999, 72, 873892.

79 P. Xie, Y. Li, Q. Hou, K. Sui, C. Liu, X. Fu, J. Zhang, V. Murugadoss, J. Fan, Y. Wang, R. Fan and Z. Guo, J. Mater. Chem. C, 2020, 8, 3029-3039.

80 S. Yoshimoto, F. Ohashi and T. Kameyama, Macromol. Rapid Commun., 2004, 25, 1687-1691. 